\title{
Continuous subadditive processes and formulae for Lyapunov characteristic exponents
}

\author{
by Wojciech SŁomczyński (Kraków)
}

\begin{abstract}
Asymptotic properties of various semidynamical systems can be examined by means of continuous subadditive processes. To investigate such processes we consider different types of exponents: characteristic, central, singular and global exponents and we study their properties. We derive formulae for central and singular exponents and show that they provide upper bounds for characteristic exponents. The concept of conjugate processes introduced in this paper allows us to find lower bounds for characteristic exponents. We also give applications to continuous cocycles.
\end{abstract}

0. Introduction. Let $\theta: M \rightarrow M$ be a diffeomorphism of a compact $d$-dimensional manifold $M$. Then the Lyapunov characteristic exponent of a vector $v$ at a point $x \in M$ is defined as the upper limit of the sequence $n^{-1} \log \left\|D_{x} \theta^{n}(v)\right\|$ as $n \rightarrow \infty$. It is easy to show that varying $v$ with $x$ fixed we obtain at most $d$ different numbers which form the so-called Lyapunov spectrum at $x$ [34]. Its maximal element (the top Lyapunov exponent) is equal to the upper limit of $n^{-1} \log \left\|D_{x} \theta^{n}\right\|$ as $n \rightarrow \infty$ [24]. Since 1968, when Oseledec proved his Multiplicative Ergodic Theorem [34], the characteristic Lyapunov exponents have become one of the basic tools in the study of smooth dynamical systems. The Oseledec theorem says, among other things, that if $\mu$ is an ergodic measure for $\theta$ then for $\mu$-almost every $x \in M$ the Lyapunov spectrum is the same and the Lyapunov exponents are strict, i.e., the upper limits in their definition can be replaced by limits. This allows us to speak of the Lyapunov exponents of a fixed ergodic measure $\mu$.

Only in case when $\mu$ is absolutely continuous with respect to the Lebesgue measure on $M$, the Oseledec theorem provides precise information on the Lyapunov exponents at the points of a "large" subset of $M$. The situation is quite different for dissipative systems where each invariant measure is singular. This leads to questions which are the starting point of this paper:

1991 Mathematics Subject Classification: 34D08, 58F11.

Key words and phrases: Lyapunov exponents, subadditive processes, invariant measures, cocycles. 
1. How in general do the Lyapunov exponents depend on the initial point of the phase space with respect to which they are calculated?

2. How are they connected with the Lyapunov exponents of ergodic measures on $M$ ?

3. When do strict exponents exist?

Similar questions already appeared in the work of Oseledec [34].

These questions seem to have a practical meaning. In recent years many papers were devoted to the computation of Lyapunov exponents by numerical methods or in physical experiments ([3], [4], [13], [16], [19], [41], [47]). The calculated exponents are a priori related to the choice of the initial point of the trajectory. It would be interesting to find the relation of the computed quantities to those coming from the Oseledec theorem (see also remarks in [24, Section 10(A)]). In some cases the experiments seem to support the conjecture that the Lyapunov spectrum does not depend on the choice of (almost every) initial point ([1], [3], [16], [21], [41]).

An answer to Questions 1-3 may also involve one of the assumptions of the Kaplan-Yorke conjectures. The fact that strict characteristic exponents exist and the Lyapunov spectrum is the same for almost all initial points with respect to the Lebesgue measure on $M$ is concerned with the conjecture on the equality of the Lyapunov dimension and probabilistic dimension of strong attractors, as stated in [17], [18] (the statement in [2] is slightly different). Another conjecture says that if all initial points in some neighbourhood of a strong attractor yield the same Lyapunov spectrum then the Lyapunov dimension of this attractor is typically equal to its capacity [17], [18].

It seems that the answer to Questions 1-3 for the top Lyapunov exponent is the following. For many smooth dynamical systems the upper limit of $n^{-1} \log \left\|D_{x} \theta^{n}\right\|$ as $n \rightarrow \infty$ is equal to the supremum of the top Lyapunov exponents of measures which are accumulation points of the sequence $\left\{n^{-1} \sum_{i=0}^{n-1} \delta_{\theta^{i} x}\right\}_{n \in \mathbb{N}}$, and the lower limit is equal to the corresponding infimum (we call such $x$ normal). In some special cases the sequence $n^{-1} \log \left\|D_{x} \theta^{n}\right\|$ converges to the supremum of the top Lyapunov exponents of measures with supports in the closure of the trajectory of $x$. In both situations we get precise information about the top Lyapunov exponents. Let us mention that interesting results in this direction were obtained in the generic case by Mañé [32] and Millonshchikov [33] by different methods. We can also obtain information about smaller exponents by considering so-called wedge product flows [24]. If the dissipative ergodic theorem [6] holds for a given smooth dynamical system (as e.g. for Axiom A dynamical systems [5] and for the Lorenz attractor [7]) and the above conjecture on Lyapunov exponents is true then the assumptions of the Kaplan-Yorke conjecture are satisfied. 
In this paper we provide a general method of dealing with the problems mentioned above based on the notion of continuous subadditive process. Such processes appear naturally in the theory of Lyapunov exponents. Define $\Phi: M \times \mathbb{N} \rightarrow M(d)$, where $M(d)$ denotes the space of all $d \times d$ matrices, by $\Phi(x, n)=D_{x} \theta^{n}$ for $x \in X$ and $n \in \mathbb{N}$; then $\Phi$ is a continuous cocycle over $\theta$ (see Definition 2.2). Further, define $g_{n}: M \rightarrow \mathbb{R} \cup\{-\infty\}$ by $g_{n}(x)=\log \|\Phi(x, n)\|$ for $x \in M$ and $n \in \mathbb{N}$; then the sequence $\left\{g_{n}\right\}_{n \in \mathbb{N}}$ is a continuous subadditive process (see Definition 2.1 ). Hence the study of Lyapunov exponents reduces to examining the convergence of $\left\{g_{n} / n\right\}_{n \in \mathbb{N}}$. The idea of applying subadditive processes to the study of Lyapunov exponents is not new. For the first time it appeared in Ranghunathan-Ruelle's proof of the Oseledec theorem [36], [37]. There are three reasons why we use it in the present paper.

Firstly, the obtained results can be applied not only to smooth dynamical systems on finite-dimensional manifolds but also in all the cases where the existence of Lyapunov exponents follows from Kingman's subadditive ergodic theorem. Such situations are encountered in semidynamical systems in Hilbert spaces [38], stochastic linear differential equations [24], stochastic dynamical systems [9], topological dynamical systems in metric spaces [25], random transformations [26] and one-dimensional cellular automata [40] $\left({ }^{1}\right)$.

Secondly, this method allows us to get generalizations and uniform proofs of various results obtained previously by Bylov et al. [8], Eden [14], [15], Johnson et al. [24], Thieullen [44], Walters [46] and others. We can see that subadditivity plays a crucial role in the study of Lyapunov exponents.

Thirdly, our method leads to new results, even in the case of smooth dynamical systems. Let us mention the formulae for central and singular exponents (Theorems 4.1, 4.5 and 5.17) which indicate a way for the numerical computation of the Lyapunov exponents of invariant measures.

To study various properties of continuous subadditive processes we generalize some old definitions including those of central, singular and global exponents (Definition 2.4) and we introduce a new notion of conjugate processes (Definition 5.1), which serves here as a basic tool.

This paper is organized as follows:

In $\S 1$ we collect some basic facts concerning semidynamical systems, invariant measures and subadditive processes which will be needed in the sequel. Also, we state a simple topological lemma which will be used frequently.

$\left({ }^{1}\right)$ Added in proof. Recently, R. Vilela Mendes used the Kingman theorem in his construction of the quantum characteristic exponents (Phys. Lett. A 187 (1994), 299$301)$. 
In $\S 2$ we define continuous subadditive processes. In addition to the commonly used characteristic exponents we introduce central, singular and global exponents, central numbers and functions. These notions allow us to relate the exponents calculated at a point with those coming from invariant measures.

We devote $\S 3$ to the study of relations between various types of exponents. The main result (Theorem 3.2) says that central and singular exponents provide upper bounds for characteristic exponents. An essential step in the proof is an inequality for continuous subadditive processes (Proposition 3.1), which is also used in the next sections.

In $\S 4$ we present two methods of calculating central, singular and global exponents. The first one allows us to find the value of an exponent from the behaviour of the continuous subadditive process on the trajectory of the given point and hence it can have a practical meaning. The second method uses the notion of central functions and numbers.

In $\S 5$ we introduce the concept of conjugate processes. We use it to obtain the invariance of characteristic exponents, lower bounds for these exponents, and equalities between characteristic and central or singular exponents. Also, we give some formulae for central and singular exponents.

In $\S 6$ we show how one can derive the continuous parameter versions of the results obtained in the preceding sections.

In this paper we deal with pointwise convergence of the sequence $\left\{g_{n} / n\right\}_{n \in \mathbb{N}}$. Uniform convergence is treated in [42]. In that paper we give several versions of the mean subadditive ergodic theorem in the space of continuous functions on a compact set.

The material of this article forms a part of the author's Ph.D. thesis (Jagiellonian University, 1990).

\section{Preliminaries}

A. Semidynamical systems. Throughout $X$ will denote a separable metric space, $\theta$ a continuous map from $X$ to $X$ and $\Theta:=\left\{\theta^{n}: n \in \mathbb{N}\right\}\left(^{2}\right)$ the discrete semidynamical system generated by $\theta$. We write $B(X)$ for the Banach space of all bounded real-valued continuous functions on $X$ with the norm $\|f\|:=\sup \{|f(x)|: x \in X\}$ and $C(X)$ for the space of all real-valued continuous functions on $X$.

We say that a set $A \subset X$ is (positively) invariant if $\theta(A) \subset A$, and that a function $f: X \rightarrow \mathbb{R} \cup\{-\infty\}$ is invariant if $f(\theta x)=f(x)$ for all $x \in X$.

For $x \in X$ the sets $\gamma^{+}(x):=\left\{\theta^{n}(x): n \in \mathbb{N}\right\}, H^{+}(x):=\operatorname{cl}\left(\gamma^{+}(x)\right)$ and $L^{+}(x):=\left\{y \in X: \theta^{n_{k}}(x) \rightarrow y(k \rightarrow \infty)\right.$ for some subsequence $\left(n_{k}\right)_{k \in \mathbb{N}}$

$\left({ }^{2}\right)$ We use $\mathbb{N}$ to denote both $\{1,2, \ldots\}$ and $\{0,1,2, \ldots\}$, depending on the context. 
of $\mathbb{N}\}$ are called the positive orbit through $x$, positive hull and positive limit set (or $\omega$-limit set) of $x$ respectively. The following lemma will be useful.

Lemma 1.1 ([39]). For every $x \in X$,

(i) $L^{+}(x)$ and $H^{+}(x)$ are closed and invariant,

(ii) $L^{+}\left(\theta^{n} x\right)=L^{+}(x)$ for all $n \in \mathbb{N}$, and

(iii) $L^{+}(x)=\bigcap_{k \in \mathbb{N}} H^{+}\left(\theta^{k} x\right)$.

General assumption: In this paper we only consider semidynamical systems that are Lagrange stable, i.e., $H^{+}(x)$ is compact for every $x \in X$.

Note that if a system is Lagrange stable then $L^{+}(x)$ is also compact for every $x \in X$.

B. Space of measures. We denote by $\mathcal{M}(X)$ the space of all Borel probability measures on $X$; we refer to them briefly as measures. For each $x \in X$ we denote by $\delta_{x}$ the Dirac measure at $x$. Let $\mu \in \mathcal{M}(X)$. The smallest closed set $C$ such that $\mu(C)=1$ is called the support of $\mu$ and denoted by supp $\mu$. If $A \subset X$ then we write $\mathcal{M}(X, A)$, or $\mathcal{M}(A)$ when no confusion can arise, for the set of all measures with support contained in $A$.

The well-known theorem of Alexandrov says that $B^{*}(X)$, the dual of $B(X)$, is isomorphic to the space of all finitely additive regular set functions on the Borel $\sigma$-field of $X$ [12]. Each measure on $X$ is regular so we can treat $\mathcal{M}(X)$ as a subset of $B^{*}(X)$ and endow it with the $w^{*}$-topology relative to $\mathcal{M}(X)$. This means that a net $\left\{\mu_{\alpha}\right\} \subset \mathcal{M}(X)$ converges to $\mu \in \mathcal{M}(X)$ if and only if $\int f d \mu_{\alpha}$ converges to $\int f d \mu$ for all $f \in B(X)$. The Alexandrov theorem also implies that if $\mu$ and $\nu$ are measures on $X$, then $\mu=\nu$ if and only if $\int f d \mu=\int f d \nu$ for every $f \in B(X)$. The topological space $\mathcal{M}(X)$ can be given a separable metric [35].

The following lemma is easy to prove.

Lemma 1.2. Let $A$ be a closed subset of $X$. Then $\mu \in \mathcal{M}(X, A)$ if and only if $\mu(A)=1$. If $A$ is compact then so is $\mathcal{M}(X, A)$.

Remark. Assume that $A$ is a compact subset of $X,\left\{\mu_{\alpha}\right\}$ is a net in $\mathcal{M}(A)$ and $\mu \in \mathcal{M}(A)$. If $\left\{\mu_{\alpha}\right\}$ converges to $\mu$ then $\int f d \mu_{\alpha}$ converges to $\int f d \mu$ for every continuous, not necessarily bounded, function $f: X \rightarrow \mathbb{R}$. This can be proved by considering first the function $\left.f\right|_{A}$, and then its continuous and bounded extension coming from the Tietze theorem.

C. Invariant measures. Let $\mu \in \mathcal{M}(X)$ and let $\Theta$ be a discrete semidynamical system on $X$. We say that $\mu$ is invariant if $\theta \mu=\mu$, where $\theta \mu$ is the measure on $X$ given by $\theta \mu(A)=\mu\left(\theta^{-1}(A)\right)$, and denote by $\mathcal{M}^{\Theta}(X)$ the set of all invariant measures on $X$. If $A \subset X$ we write $\mathcal{M}^{\Theta}(A)$ for $\mathcal{M}^{\Theta}(X) \cap \mathcal{M}(X, A)$. We say that $A \subset X$ is of total measure if $\mu(A)=1$ for 
all $\mu \in \mathcal{M}^{\Theta}(X)$. We call an invariant measure ergodic if each measurable invariant set has either measure 0 or 1 . It is easy to show that $\mathcal{M}^{\Theta}(X)$ is a closed and convex subset of $\mathcal{M}(X)$.

Now we introduce two set-valued functions which will play an important role in the sequel.

Definition 1.3. Let $x \in X$. We define

$V(x):=\left\{\mu \in \mathcal{M}(X): \mu\right.$ is an accumulation point of $\left.\left\{n^{-1} \sum_{i=0}^{n-1} \delta_{\theta^{i} x}\right\}_{n \in \mathbb{N}}\right\}$,

$S(x):=\mathcal{M}^{\Theta}\left(H^{+}(x)\right)$.

Lemma 1.4. If $A \subset X$ is compact and invariant, $\left(x_{n}\right)_{n \in \mathbb{N}}$ is a sequence in $A$ and $\mu$ is an accumulation point of $\left\{n^{-1} \sum_{i=0}^{n-1} \delta_{\theta^{i} x_{n}}\right\}_{n \in \mathbb{N}}$, then $\mu \in \mathcal{M}^{\Theta}(A)$.

Proof. Let $\mu$ be the limit of the sequence $\left\{n_{k}^{-1} \sum_{i=0}^{n_{k}-1} \delta_{\theta^{i} y_{k}}\right\}_{k \in \mathbb{N}}$, where $y_{k}=x_{n_{k}}$ for $k \in \mathbb{N}$. As all the elements of that sequence belong to $\mathcal{M}(A)$ so does $\mu$ by Lemma 1.2. Thus it is enough to prove that $\mu$ is invariant. By the Alexandrov theorem, it is sufficient to show that $\int f d \mu=\int f d \theta \mu$ for every $f \in B(X)$. We have

$$
\begin{aligned}
\int f d \theta \mu & =\int f \circ \theta d \mu=\lim _{k \rightarrow+\infty} n_{k}^{-1} \sum_{i=0}^{n_{k}-1} f\left(\theta^{i+1} y_{k}\right) \\
& =\lim _{k \rightarrow+\infty} n_{k}^{-1}\left\{\sum_{i=0}^{n_{k}-1} f\left(\theta^{i} y_{k}\right)+f\left(\theta^{n_{k}} y_{k}\right)-f\left(y_{k}\right)\right\} .
\end{aligned}
$$

The first term converges to $\int f d \mu$ and the last two to 0 since $\mid n_{k}^{-1}\left(f\left(\theta^{n_{k}} y_{k}\right)-\right.$ $\left.f\left(y_{k}\right)\right) \mid \leq 2 n_{k}^{-1}\|f\|_{A}$.

R e m ark. The above proof shows that if the assumptions of Lemma 1.4 are satisfied then $\int f d \mu=\int f d \theta \mu$ for every continuous, not necessarily bounded, function $f: X \rightarrow \mathbb{R}$.

Proposition 1.5. For every $x \in X$,

(i) $V(x) \subset S(x)$,

(ii) $V(x)$ and $S(x)$ are non-empty compact subsets of $\mathcal{M}(X)$,

(iii) $S(x)=\mathcal{M}^{\Theta}\left(L^{+}(x)\right)$,

(iv) $V\left(\theta^{k} x\right)=V(x)$ and $S\left(\theta^{k} x\right)=S(x)$ for each $k \in \mathbb{N}$.

Pro of. (i) In Lemma 1.4, take $A=H^{+}(x)$ and $x_{n}=x$ for all $n \in \mathbb{N}$.

(ii) By Lemma 1.2, $S(x)$ is compact as the intersection of the closed set $\mathcal{M}^{\Theta}(X)$ and the compact set $\mathcal{M}\left(H^{+}(x)\right)$. Next, $V(x)$ is closed and nonempty, being the set of all accumulation points of a sequence contained in the compact set $\mathcal{M}\left(H^{+}(x)\right)$. Hence $S(x)$ is non-empty and $V(x)$ is compact. 
(iii) Let $\mu \in \mathcal{M}^{\Theta}(X)$. By Lemma 1.2, it is enough to show that $\mu\left(H^{+}(x)\right)$ $=1$ if and only if $\mu\left(L^{+}(x)\right)=1$. Since $H^{+}(x) \subset \theta^{-k}\left(H^{+}\left(\theta^{k} x\right)\right)$ for each $k \in \mathbb{N}$, Lemma 1.1(iii) gives

$$
\mu\left(L^{+}(x)\right)=\mu\left(\bigcap_{k \in \mathbb{N}} H^{+}\left(\theta^{k} x\right)\right)=\lim _{k \rightarrow+\infty} \mu\left(H^{+}\left(\theta^{k} x\right)\right) \geq \mu\left(H^{+}(x)\right),
$$

which proves (iii).

(iv) Let $x \in X, k \in \mathbb{N}$. To prove $V(x)=V\left(\theta^{k} x\right)$ it is sufficient to show that

$$
\lim _{u \rightarrow+\infty} n_{u}^{-1} \sum_{i=0}^{n_{u}-1}\left(\delta_{\theta^{i} x}-\delta_{\theta^{i+k} x}\right)=0
$$

for every subsequence $\left(n_{u}\right)_{u \in \mathbb{N}}$ of $\mathbb{N}$. We have

$$
\begin{aligned}
\lim _{u \rightarrow+\infty}\left|n_{u}^{-1} \sum_{i=0}^{n_{u}-1}\left(f\left(\theta^{i} x\right)-f\left(\theta^{i+k} x\right)\right)\right| & =\lim _{u \rightarrow+\infty}\left|n_{u}^{-1} \sum_{i=0}^{k-1}\left(f\left(\theta^{i} x\right)-f\left(\theta^{i+n_{u}} x\right)\right)\right| \\
& \leq \lim _{u \rightarrow+\infty} n_{u}^{-1} \sum_{i=0}^{k-1} 2\|f\|_{H^{+}(x)}=0
\end{aligned}
$$

for all $f \in B(X)$, as required. To show the second equality, observe that by (iii) and Lemma 1.1(ii), we have $S(x)=\mathcal{M}^{\Theta}\left(L^{+}(x)\right)=\mathcal{M}^{\Theta}\left(L^{+}\left(\theta^{k} x\right)\right)=$ $S\left(\theta^{k} x\right)$.

The next proposition connects the limits of the ergodic averages with the sets of measures introduced above. In the proof we use the following classical lemma.

LEMma 1.6 (the subadditive lemma). Let $\left(a_{n}\right)_{n \in \mathbb{N}}$ be a subadditive sequence in $\mathbb{R} \cup\{-\infty\}$ (i.e. $a_{n+k} \leq a_{n}+a_{k}$ for all $k, n \in \mathbb{N}$ ). Then $a_{n} / n$ converges to $\inf \left\{a_{n} / n: n \in \mathbb{N}\right\}$.

Proposition 1.7. Let $x \in X$, let $A$ be a non-empty compact invariant set, and let $g \in C(X)$. Then

(i) $\limsup _{n \rightarrow+\infty} n^{-1} \sum_{i=0}^{n-1} g\left(\theta^{i} x\right)=\sup \left\{\int g d \mu: \mu \in V(x)\right\}$,

$$
\liminf _{n \rightarrow+\infty} n^{-1} \sum_{i=0}^{n-1} g\left(\theta^{i} x\right)=\inf \left\{\int g d \mu: \mu \in V(x)\right\}
$$

(ii) $\lim _{n \rightarrow+\infty} \max _{A} n^{-1} \sum_{i=0}^{n-1} g \circ \theta^{i}=\inf _{n \in \mathbb{N}} \max _{A} n^{-1} \sum_{i=0}^{n-1} g \circ \theta^{i}$

$$
=\sup \left\{\int g d \mu: \mu \in \mathcal{M}^{\Theta}(A)\right\} \text {, }
$$


(iii) $\quad \lim _{n \rightarrow+\infty} \sup _{j \in \mathbb{N}} n^{-1} \sum_{i=0}^{n-1} g\left(\theta^{i+j} x\right)=\inf _{n \in \mathbb{N}} \sup _{j \in \mathbb{N}} n^{-1} \sum_{i=0}^{n-1} g\left(\theta^{i+j} x\right)$

$$
=\sup \left\{\int g d \mu: \mu \in S(x)\right\},
$$

(iv) in (iii) "sup $\operatorname{seN}_{j \in \mathbb{N}}$ can be replaced by "lim $\sup _{j \rightarrow+\infty}$ ".

Proof. (i) Put $l:=\lim \sup _{n \rightarrow+\infty} n^{-1} \sum_{i=0}^{n-1} g\left(\theta^{i} x\right)$ and $s:=\sup \left\{\int g d \mu\right.$ : $\mu \in V(x)\}$. Let $\mu \in V(x)$. Then there exists a subsequence $\left(n_{k}\right)_{k \in \mathbb{N}}$ of $\mathbb{N}$ such that $\mu=\lim _{k \rightarrow+\infty} n_{k}^{-1} \sum_{i=0}^{n_{k}-1} \delta_{\theta^{i} x}$. Hence

$$
\int g d \mu=\lim _{k \rightarrow+\infty} n_{k}^{-1} \sum_{i=0}^{n_{k}-1} g\left(\theta^{i} x\right) \leq l .
$$

Thus $s \leq l$. On the other hand, there exists a subsequence $\left(n_{u}\right)_{u \in \mathbb{N}}$ of $\mathbb{N}$ such that $l=\lim _{u \rightarrow+\infty} n_{u}^{-1} \sum_{i=0}^{n_{u}-1} g\left(\theta^{i} x\right)$. Set $\mu_{u}:=n_{u}^{-1} \sum_{i=0}^{n_{u}-1} \delta_{\theta^{i} x} \in$ $\mathcal{M}\left(H^{+}(x)\right)$ for $u \in \mathbb{N}$. By Lemma 1.2, passing to a subsequence if necessary, assume that $\left(\mu_{u}\right)_{u \in \mathbb{N}}$ converges to some $\mu \in \mathcal{M}\left(H^{+}(x)\right)$. Clearly, $\mu \in V(x)$. We have

$$
l=\lim _{u \rightarrow+\infty} n_{u}^{-1} \sum_{i=0}^{n_{u}-1} g\left(\theta^{i} x\right)=\int g d \mu \leq s,
$$

which proves the first equality of (i). Applying it to $h=-g$ we get the second equality.

(ii) First note that the sequence $a_{n}:=\max _{A} \sum_{i=0}^{n-1} g \circ \theta^{i}$ for $n \in \mathbb{N}$ is subadditive. By Lemma 1.6, $\lim _{n \rightarrow+\infty} n^{-1} a_{n}=\inf _{n \in \mathbb{N}} n^{-1} a_{n}$, which proves the first equality of (ii). Next, suppose that $\mu \in \mathcal{M}^{\Theta}(A)$. Then $\int f d \mu \leq$ $\max _{A} f$ for $f \in C(X)$. Applying this to $f_{n}:=n^{-1} \sum_{i=0}^{n-1} g \circ \theta^{i}$ for $n \in \mathbb{N}$, we get $\int g d \mu=\int f_{n} d \mu \leq n^{-1} a_{n}$. Therefore $\sup \left\{\int g d \mu: \mu \in \mathcal{M}^{\Theta}(A)\right\} \leq$ $\inf _{n \in \mathbb{N}} n^{-1} a_{n}$. To prove the reverse inequality take, for each $n \in \mathbb{N}, x_{n} \in$ $A$ such that $n^{-1} a_{n}=n^{-1} \sum_{i=0}^{n-1} g\left(\theta^{i} x_{n}\right)$. Set $\mu_{n}:=n^{-1} \sum_{i=0}^{n-1} \delta_{\theta^{i} x_{n}}$. By Lemma 1.2 we can assume that $\left(\mu_{n}\right)$ converges to some $\nu$. Then $\nu \in \mathcal{M}^{\Theta}(A)$ by Lemma 1.4. Hence

$$
\begin{aligned}
\lim _{n \rightarrow+\infty} n^{-1} a_{n} & =\lim _{n \rightarrow+\infty} n^{-1} \sum_{i=0}^{n-1} g\left(\theta^{i} x_{n}\right)=\int g d \nu \\
& \leq \sup \left\{\int g d \mu: \mu \in \mathcal{M}^{\Theta}(A)\right\},
\end{aligned}
$$

which completes the proof of (ii).

(iii) follows immediately from (ii), by taking $A:=H^{+}(x)$.

(iv) As in the proof of (ii) the first equality follows from the subadditivity 
of $\left\{\lim \sup _{j \rightarrow+\infty} \sum_{i=0}^{n-1} g\left(\theta^{i+j} x\right)\right\}_{n \in \mathbb{N}}$. From (iii) we obtain

$$
\begin{aligned}
& \lim _{n \rightarrow+\infty} \limsup _{j \rightarrow+\infty} n^{-1} \sum_{i=0}^{n-1} g\left(\theta^{i+j} x\right) \\
& \leq \lim _{n \rightarrow+\infty} \sup _{j \in \mathbb{N}} n^{-1} \sum_{i=0}^{n-1} g\left(\theta^{i+j} x\right)=\sup \left\{\int g d \mu: \mu \in S(x)\right\} .
\end{aligned}
$$

To prove the reverse inequality observe that for $\mu \in S(x)$,

$$
\begin{aligned}
\int f d \mu & \leq \sup \left\{f(z): z \in L^{+}(x)\right\}=\sup \left\{f(z): z \in \bigcap_{k \in \mathbb{N}} H^{+}\left(\theta^{k} x\right)\right\} \\
& =\inf _{k \in \mathbb{N}} \sup _{j \geq k} f\left(\theta^{j} x\right)=\limsup _{j \rightarrow+\infty} f\left(\theta^{j} x\right)
\end{aligned}
$$

for $f \in C(X)$. Applying this to $f_{n}:=n^{-1} \sum_{i=0}^{n-1} g \circ \theta^{i}$ we get the desired result.

Remark. The assertion (ii) can also be deduced from a more general result of Crauel [11, Theorem 6]. Moreover, in the proof of (iv) we could use [20, Proposition 3.9]. To make our exposition self-contained, we gave a direct proof.

We shall also consider the semidynamical systems $\Theta_{m}$ generated by $\theta^{m}$ for $m \in \mathbb{N}$. Then $V_{m}$ and $S_{m}$ will denote the corresponding set-valued maps connected with these systems.

D. Subadditive processes. The theory of measurable subadditive processes was developed by J. F. C. Kingman who proved the celebrated subadditive ergodic theorem [27]-[29]. Since then, it has been generalized in many different directions (see [30] for details). Let us now recall some basic definitions and results.

Definition 1.8 [37]. Let $(\Omega, \Sigma, \mu)$ be a probability space, let $\theta: \Omega \rightarrow \Omega$ be a measurable $\mu$-preserving map and let $\mathcal{G}:=\left(g_{n}\right)_{n \in \mathbb{N}}$ be a family of measurable functions from $\Omega$ to $\mathbb{R} \cup\{-\infty\}$. We call $\mathcal{G}$ a measurable subadditive process if the following two conditions are satisfied:

(a) (integrability) $g_{1}^{+}=\max \left(g_{1}, 0\right) \in L^{1}(\Omega, \mu)$,

(b) (subadditivity) $g_{n+k} \leq g_{n}+g_{k} \circ \theta^{n} \mu$-almost everywhere, for $n, k \in \mathbb{N}$.

We say that $\mathcal{G}$ is additive if equality holds in (b). Note that an additive process must be of the form $\sum_{i=0}^{n-1} g \circ \theta^{i}$ for some measurable $g: \Omega \rightarrow$ $\mathbb{R} \cup\{-\infty\}$.

Definition 1.9. In the above situation we define

$$
\Lambda(\mu):=\inf _{n \in \mathbb{N}} n^{-1} \int g_{n} d \mu \text {. }
$$


If we deal with more than one subadditive process we write $\Lambda_{\mathcal{G}}(\mu)$ instead of $\Lambda(\mu)$.

It is easy to deduce from (b) that the sequence $\left\{\int g_{n} d \mu\right\}_{n \in \mathbb{N}}$ is subadditive. Applying Lemma 1.6 we get

Proposition 1.10. $\Lambda(\mu)=\lim _{n \rightarrow+\infty} n^{-1} \int g_{n} d \mu$.

The following is a version of the Kingman subadditive ergodic theorem given by Ruelle [37].

THEorem 1.11 (Subadditive ergodic theorem). Let $\mathcal{G}:=\left(g_{n}\right)_{n \in \mathbb{N}}$ be a measurable subadditive process. Then there exists a measurable function $g: \Omega \rightarrow \mathbb{R} \cup\{-\infty\}$ such that

(i) $g^{+} \in L^{1}(\Omega, \mu)$,

(ii) $g$ is $\theta$-invariant,

(iii) $g=\lim _{n \rightarrow+\infty} n^{-1} g_{n} \mu$-a.e.,

(iv) $\int g d \mu=\Lambda(\mu)$.

If additionally $\theta$ is ergodic in $(\Omega, \Sigma, \mu)$, then $g=\Lambda(\mu) \mu$-a.e.

Note that if $\mathcal{G}$ is a measurable subadditive process with respect to each $\mu \in \mathcal{M}^{\Theta}(X)$ then we can choose $g$ in the above theorem independent of $\mu$.

Most of the present paper is independent of the Kingman subadditive ergodic theorem.

Proposition 1.12. Let $\mathcal{G}$ be a measurable subadditive process with respect to each $\mu \in \mathcal{M}^{\Theta}(X)$. Then

$$
\Lambda(\mu)=\int \Lambda\left(m_{x}\right) d \mu(x),
$$

where $x \mapsto m_{x}$ is the ergodic decomposition relative to $\Theta$ (see [26]).

Pro of. Let $\mu \in \mathcal{M}^{\Theta}(X)$. Then, by Theorem 1.11, we have

$$
\Lambda(\mu)=\int g d \mu=\int\left(\int g d m_{x}\right) d \mu(x)=\int \Lambda\left(m_{x}\right) d \mu(x) .
$$

E. A topological lemma. We conclude this section with the following useful lemma.

Lemma 1.13. Let $M$ be a compact topological space. If $\left\{G_{m}\right\}_{m \in \mathbb{N}}$ is a subadditive sequence of upper semicontinuous functions from $M$ to $\mathbb{R} \cup\{-\infty\}$, then

(i) $\inf _{m \in \mathbb{N}} m^{-1} G_{m}(x)=\lim _{m \rightarrow+\infty} m^{-1} G_{m}(x)=: G(x)$ for all $x \in M$,

(ii) $\inf _{x \in M} G(x)=\inf _{m \in \mathbb{N}} \inf _{x \in M} m^{-1} G_{m}(x)=\lim _{m \rightarrow+\infty} \inf _{x \in M} m^{-1} G_{m}(x)$,

(iii) $\max _{x \in M} G(x)=\inf _{m \in \mathbb{N}} \max _{x \in M} m^{-1} G_{m}(x)=\lim _{m \rightarrow+\infty} \max _{x \in M} m^{-1} G_{m}(x)$. 
Proof. (i) follows from Lemma 1.6. To prove (ii) fix $N \in \mathbb{N}$. Put $A_{N}:=$ $\max \left\{G_{j}(x): x \in M, j=0, \ldots, N-1\right\}$. For each $m \in \mathbb{N}$, there exist $k_{m} \in \mathbb{N}$ and $r_{m} \in\{0, \ldots, N-1\}$ such that $m=k_{m} N+r_{m}$. Then

$$
G_{m}(x) \leq k_{m} G_{N}(x)+G_{r_{m}}(x) \leq k_{m} G_{N}(x)+A_{N}
$$

for all $x \in M$. Hence

$$
\limsup _{m \rightarrow+\infty} \inf _{x \in M} \frac{1}{m} G_{m}(x) \leq \inf _{x \in M} \frac{1}{N} G_{N}(x) .
$$

As $N$ was arbitrary we get the desired result.

(iii) Note first that $G$ is upper semicontinuous, being the infimum of a sequence of upper semicontinuous functions. Hence $G$, as well as each $G_{n}$, attain their suprema on $M$.

The second equality follows from the subadditivity of $\max \left\{G_{m}(x): x \in\right.$ $M\}$ and Lemma 1.6. We put $\alpha:=\inf _{m \in \mathbb{N}} \max _{x \in M} m^{-1} G_{m}(x)$. Applying (i) we get $\max _{x \in M} G(x) \leq \alpha$. To prove the reverse inequality, set $B_{m}:=$ $\left\{x \in M: G_{m}(x) \geq m \alpha\right\}$ for $m \in \mathbb{N}$. Since $G_{m}$ is upper semicontinuous, $B_{m}$ is closed for each $m \in \mathbb{N}$. Now we show that $\left\{B_{m}\right\}_{m \in \mathbb{N}}$ has the finite intersection property. Let $m_{1}, \ldots, m_{k} \in \mathbb{N}$. Set $n:=m_{1} \cdot \ldots \cdot m_{k}$. Then $n^{-1} G_{n} \leq \min \left\{m_{i}^{-1} G_{m_{i}}: i=1, \ldots, k\right\}$. Hence $\emptyset \neq B_{n} \subset \bigcap_{i=1}^{k} B_{m_{i}}$. Thus there exists $x_{0} \in M$ such that $G_{m}\left(x_{0}\right) \geq m \alpha$ for each $m \in \mathbb{N}$. Hence $G\left(x_{0}\right) \geq \alpha$ and so $\max \{G(x): x \in M\} \geq \alpha$.

\section{Characteristic, central, singular and global exponents and their basic properties}

Definition 2.1. Let $\mathcal{G}:=\left(g_{n}\right)_{n \in \mathbb{N}}$ be a family of functions from $X$ to $\mathbb{R} \cup\{-\infty\}$. We say that $\mathcal{G}$ is a continuous subadditive process (c.s.p.) if the following two conditions are satisfied:

(a) (continuity) $g_{n}$ is continuous for each $n \in \mathbb{N}$,

(b) (subadditivity) $g_{n+k} \leq g_{n}+g_{k} \circ \theta^{n}$ for $n, k \in \mathbb{N}$.

We say that $\mathcal{G}$ is finite if it assumes only finite values.

It is clear that each continuous subadditive process satisfies the integrability condition from Definition 1.8 and hence it is a measurable subadditive process.

Definition 2.2. Let $M(d)$ be the space of $d \times d$ matrices and $\Phi$ a map from $X \times \mathbb{N}$ to $M(d)$. We say that $\Phi$ is a continuous cocycle if

(i) $\Phi$ is continuous,

(ii) $\Phi$ satisfies the cocycle equality: $\Phi(x, n+k)=\Phi\left(\theta^{n} x, k\right) \circ \Phi(x, n)$ for all $x \in X$ and $n, k \in \mathbb{N}$. 
In the present paper we consider continuous cocycles only, so we call them briefly cocycles. Each cocycle generates a c.s.p. in the following way:

Proposition 2.3. Let $g_{n}: X \rightarrow \mathbb{R} \cup\{-\infty\}$ be given by $g_{n}(x):=$ $\ln \|\Phi(x, n)\|$ for $x \in X$ and $n \in \mathbb{N}$, where \|\| is an arbitrary Banach algebra norm in $M(d)$ (we put $\ln 0:=-\infty)$. Then $\left(g_{n}\right)_{n \in \mathbb{N}}$ is a c.s.p.

The proof is simple and is omitted.

The main subject of our work is the study of the convergence of the sequence $\left\{n^{-1} g_{n}\right\}_{n \in \mathbb{N}}$ for a c.s.p. $\left(g_{n}\right)_{n \in \mathbb{N}}$. Now we introduce the quantities which will be used to estimate the limit. We call them exponents meaning the applications to the cocycles.

Definition 2.4. Suppose $\mathcal{G}:=\left(g_{n}\right)_{n \in \mathbb{N}}$ is a c.s.p., $x \in X$ and $A$ is a non-empty closed invariant subset of $X$. We call

$$
\begin{aligned}
& \underline{\Lambda}(x):=\liminf _{n \rightarrow+\infty} n^{-1} g_{n}(x) \text { - the lower characteristic exponent at } x, \\
& \bar{\Lambda}(x):=\limsup _{n \rightarrow+\infty} n^{-1} g_{n}(x) \text { - the upper characteristic exponent at } x, \\
& \underline{\Omega}(x):=\inf \{\Lambda(\mu): \mu \in V(x)\} \text { - the lower central exponent at } x, \\
& \bar{\Omega}(x):=\sup \{\Lambda(\mu): \mu \in V(x)\} \text { - the upper central exponent at } x, \\
& \Sigma(x):=\sup \{\Lambda(\mu): \mu \in S(x)\} \text { - the singular exponent at } x, \\
& \Sigma(A):=\sup \left\{\Lambda(\mu): \mu \in \mathcal{M}^{\Theta}(A)\right\} \text { - the global exponent of } A .
\end{aligned}
$$

Note that the singular exponent at $x$ is equal to the global exponent of the set $H^{+}(x)$. If the lower characteristic or central exponent is equal to the corresponding upper one, we denote their common value by $\Lambda(x)$ or $\Omega(x)$, respectively, and we say that the exponent is strict.

The class of functions we now define provides upper bounds for characteristic exponents (see Section 4).

Definition 2.5. Let $\mathcal{G}=\left(g_{n}\right)_{n \in \mathbb{N}}$ be a c.s.p. and let $R: \mathbb{N} \times X \rightarrow \mathbb{R}$. We say that $R$ is a central function if for each $\varepsilon>0$ and $x \in X$ there exists $D \in \mathbb{R}$ such that

$$
g_{n}\left(\theta^{k} x\right) \leq D+\sum_{i=k}^{n+k-1}(R(i, x)+\varepsilon) \quad \text { for all } n, k \in \mathbb{N} .
$$

We denote the set of all central functions by $\mathcal{R}$. We write $\mathcal{R}_{0}$ for the subset of $\mathcal{R}$ consisting of all central functions $R$ such that $R(\cdot, x)$ is constant for each $x \in X$. Let $r \in \mathbb{R}$. We say that $r$ is a central number of the set $A$ if for each $\varepsilon>0$ there exists $D \in \mathbb{R}$ such that

$$
g_{n}(x) \leq D+n(r+\varepsilon) \quad \text { for all } n \in \mathbb{N} \text { and } x \in A .
$$


The set of all central numbers of $A$ is denoted by $\mathcal{R}_{A}$. Note that if $r(x)$ is a central number of $H^{+}(x)$ for each $x \in X$, then the function $R$ defined by $R(n, x):=r(x)$ for $n \in \mathbb{N}$ and $x \in X$ belongs to $\mathcal{R}_{0}$.

Now we describe the approximation method which allows us to reduce the study of c.s.p.'s to the finite case.

Lemma 2.6. Let $\mathcal{G}:=\left(g_{n}\right)_{n \in \mathbb{N}}$ be a c.s.p. For each $N \in \mathbb{N}$ define

$$
g_{n}^{(N)}(x):=\max \left(g_{n}(x),-n N\right) \quad \text { for all } x \in X \text { and } n \in \mathbb{N} \text {. }
$$

Then

(i) $\left(g_{n}^{(N)}\right)_{n \in \mathbb{N}}$ is a finite c.s.p. (we mark its exponents by the superscript

(ii) $\int g_{n}^{(N)} d \mu \downarrow \int g_{n} d \mu(N \rightarrow+\infty)$ for all $\mu \in \mathcal{M}(X)$ and $n \in \mathbb{N}$,

(iii) $\Lambda(\mu)=\inf _{N \in \mathbb{N}} \Lambda^{(N)}(\mu)$ for each $\mu \in \mathcal{M}^{\Theta}(X)$,

(iv) $\underline{\Lambda}(x)=\inf _{N \in \mathbb{N}} \underline{\Lambda}^{(N)}(x)$ and $\bar{\Lambda}(x)=\inf _{N \in \mathbb{N}} \bar{\Lambda}^{(N)}(x)$ for each $x \in X$,

(v) $\underline{\Omega}(x)=\inf _{N \in \mathbb{N}} \underline{\Omega}^{(N)}(x)$ and $\bar{\Omega}(x)=\inf _{N \in \mathbb{N}} \bar{\Omega}^{(N)}(x)$ for each $x \in X$,

(vi) $\Sigma(x)=\inf _{N \in \mathbb{N}} \Sigma^{(N)}(x)$ for each $x \in X$,

(vii) $\Sigma(A)=\inf _{N \in \mathbb{N}} \Sigma^{(N)}(A)$ for each non-empty compact invariant set $A$.

Proof. (i) is evident.

(ii) Let $\mu \in \mathcal{M}(X)$ and $n \in \mathbb{N}$. We have

$$
\int g_{n}^{(N)} d \mu=\int g_{n}^{(N)+} d \mu-\int g_{n}^{(N)-} d \mu=\int g_{n}^{+} d \mu-\int g_{n}^{(N)-} d \mu .
$$

Now observe that $\int g_{n}^{(N)-} d \mu \uparrow \int g_{n}^{-} d \mu(N \rightarrow+\infty)$ (by the Lebesgue monotone convergence theorem).

(iii) Let $\mu \in \mathcal{M}^{\Theta}(X)$. Then by (ii) we have

$$
\begin{aligned}
\Lambda(\mu) & =\inf _{n \in \mathbb{N}} \int n^{-1} g_{n} d \mu=\inf _{n \in \mathbb{N}} \inf _{N \in \mathbb{N}} \int n^{-1} g_{n}^{(N)} d \mu \\
& =\inf _{N \in \mathbb{N}} \inf _{n \in \mathbb{N}} \int n^{-1} g_{n}^{(N)} d \mu=\inf _{N \in \mathbb{N}} \Lambda^{(N)}(\mu) .
\end{aligned}
$$

(iv) For each $N \in \mathbb{N}$, the function $c_{N}: \mathbb{R} \rightarrow \mathbb{R}$ defined by $c_{N}(t):=$ $\max (t,-N)$ for $t \in \mathbb{R}$ is continuous and non-decreasing. Hence

$$
\underline{\Lambda}^{(N)}(x)=\liminf _{n \rightarrow+\infty} c_{N}\left(n^{-1} g_{n}(x)\right)=c_{N}\left(\liminf _{n \rightarrow+\infty} n^{-1} g_{n}(x)\right)=c_{N}(\underline{\Lambda}(x)) .
$$

This implies the first equality of (iv). The second is proved analogously.

(v) Let now $M$ be an arbitrary compact subset of $\mathcal{M}^{\Theta}(X)$. Let $N \in \mathbb{N}$. Define $G_{N}: M \rightarrow \mathbb{R}$ by

$$
G_{N}(\mu):=N \Lambda^{(N)}(\mu) \quad \text { for } \mu \in M .
$$

It is easy to check that $\left(G_{N}\right)_{N \in \mathbb{N}}$ satisfies the assumptions of Lemma 1.13. Setting $M$ equal in turn to $V(x), S(x)$ and $\mathcal{M}^{\Theta}(X)$, then applying (ii) and Lemma 1.13(iii), we obtain (v),(vi), and (vii), respectively. 
Proposition 2.7. Suppose $\mathcal{G}:=\left(g_{n}\right)_{n \in \mathbb{N}}$ is a c.s.p., $x \in X$ and $A$ is a non-empty compact invariant subset of $X$. Then

(i) in the definitions of central, singular and global exponents, "sup" can be replaced by "max",

(ii) $\underline{\Omega}(x)=\inf _{m \in \mathbb{N}} \inf \left\{m^{-1} \int g_{m} d \mu: \mu \in V(x)\right\}$,

(iii) $\bar{\Omega}(x)=\inf _{m \in \mathbb{N}} \max \left\{m^{-1} \int g_{m} d \mu: \mu \in V(x)\right\}$,

(iv) $\Sigma(x)=\inf _{m \in \mathbb{N}} \max \left\{m^{-1} \int g_{m} d \mu: \mu \in S(x)\right\}$,

(v) $\Sigma(A)=\inf _{m \in \mathbb{N}} \max \left\{m^{-1} \int g_{m} d \mu: \mu \in \mathcal{M}^{\Theta}(A)\right\}$,

(vi) in the above formulae, " $\inf _{m \in \mathbb{N}}$ " can be replaced by " $\lim _{m \rightarrow+\infty}$ ".

(vii) $\Sigma(A)=\max \left\{\Lambda(\nu): \nu \in \mathcal{M}^{\Theta}(A), \nu\right.$ is ergodic $\}$.

Proof. Let $M$ be a compact subset of $\mathcal{M}^{\Theta}(X)$. Consider $G_{m}: M \rightarrow$ $\mathbb{R} \cup\{-\infty\}$ defined by

$$
G_{m}(\mu):=\int g_{m} d \mu \quad \text { for } \mu \in M \text { and } m \in \mathbb{N} .
$$

If $\mathcal{G}$ is a finite c.s.p. then each $G_{m}$ is continuous. In general, $G_{m}$ is upper semicontinuous, by Lemma 2.6(ii). Hence $G_{m}$ attains its maximum on $M$. It is easy to show that the sequence $\left(G_{m}\right)_{m \in \mathbb{N}}$ is subadditive. By Lemma 1.13 we get

and

$$
\begin{aligned}
\inf \{\Lambda(\mu): \mu \in M\} & =\inf _{m \in \mathbb{N}} \inf \left\{m^{-1} \int g_{m} d \mu: \mu \in M\right\} \\
& =\lim _{m \rightarrow+\infty} \inf \left\{m^{-1} \int g_{m} d \mu: \mu \in M\right\}
\end{aligned}
$$

$$
\begin{aligned}
\max \{\Lambda(\mu): \mu \in M\} & =\inf _{m \in \mathbb{N}} \max \left\{m^{-1} \int g_{m} d \mu: \mu \in M\right\} \\
& =\lim _{m \rightarrow+\infty} \max \left\{m^{-1} \int g_{m} d \mu: \mu \in M\right\} .
\end{aligned}
$$

Taking $M$ equal in turn to $V(x), S(x)$ and $\mathcal{M}^{\Theta}(A)$ we get (i)-(vi).

Now we prove (vii). It follows from (i) that there exists $\mu \in \mathcal{M}^{\Theta}(A)$ such that $\Sigma(A)=\Lambda(\mu)$. From Proposition 1.12 we have $\Sigma(A)=\int_{A} \Lambda\left(m_{x}\right) d \mu(x)$, where $x \mapsto m_{x}$ is the ergodic decomposition connected with $\Theta$. Moreover, $\Lambda\left(m_{x}\right) \leq \Sigma(A)$ for all $x \in A$. Hence $\Lambda\left(m_{x}\right)=\Sigma(A), m$-almost everywhere on $A$, which gives (vii).

TheOREM 2.8 (on invariance of exponents). Let $\mathcal{G}:=\left(g_{n}\right)_{n \in \mathbb{N}}$ be a c.s.p., $x \in X$ and $n \in \mathbb{N}$. Then

(i) $\underline{\Lambda}(x) \leq \underline{\Lambda}\left(\theta^{n} x\right), \bar{\Lambda}(x) \leq \bar{\Lambda}\left(\theta^{n} x\right)$,

(ii) $\underline{\Omega}(x)=\underline{\Omega}\left(\theta^{n} x\right), \bar{\Omega}(x)=\bar{\Omega}\left(\theta^{n} x\right)$,

(iii) $\Sigma(x)=\Sigma\left(\theta^{n} x\right)$. 
Proof. We have

$$
\begin{aligned}
\underline{\Lambda}(x) & =\liminf _{k \rightarrow+\infty} k^{-1} g_{k}(x)=\liminf _{k \rightarrow+\infty}(n+k)^{-1} g_{n+k}(x) \\
& \leq \liminf _{k \rightarrow+\infty}\left(k^{-1} g_{k}\left(\theta^{n} x\right)+k^{-1} g_{n}(x)\right)=\underline{\Lambda}\left(\theta^{n} x\right) .
\end{aligned}
$$

The proof of $\bar{\Lambda}(x) \leq \bar{\Lambda}\left(\theta^{n} x\right)$ is analogous. The assertions (ii) and (iii) follow from Proposition 1.5(iv).

The following simple example shows that the inequalities in (i) may be strict, i.e., characteristic exponents need not be invariant.

EXAMPLE 2.9. Let $X$ be a compact space and $\Theta$ a semidynamical system in $X$ with an asymptotically stable fixed point $x_{0} \in X$. If $W: X \rightarrow \mathbb{R}^{+}$is a strong Lyapunov function (i.e. $W$ is continuous, $W\left(x_{0}\right)=0, W(x)>0$ for $x \neq x_{0}, W(x)>W\left(\theta^{k} x\right)$ for $\left.x \neq x_{0}, k \in \mathbb{N}\right)$ then $g_{n}(x)=-n W(x)$ for $x \in X, n \in \mathbb{N}$ defines a c.s.p. with $\Lambda(x)<\Lambda\left(\theta^{k} x\right)$ for $x \neq x_{0}, k \in \mathbb{N}$.

In Section 5 we give a useful sufficient condition for the invariance of characteristic exponents.

Let $\mathcal{G}$ be a c.s.p. and $x \in X$. We denote by $G(x)$ the set of all accumulation points of the sequence $\left\{n^{-1} g_{n}(x)\right\}_{n \in \mathbb{N}}$.

Proposition 2.10. Let $\mathcal{G}:=\left(g_{n}\right)_{n \in \mathbb{N}}$ be a c.s.p. and $x \in X$. Then $G(x)=[\underline{\Lambda}(x), \bar{\Lambda}(x)]$ and $G(x) \subset \mathbb{R} \cup\{-\infty\}$.

Proof. It is clear that $G(x)$ is closed. It is enough to show that it is connected and does not contain $+\infty$. First assume that $g_{k}(x)=-\infty$ for some $k \in \mathbb{N}$. Then $g_{n}(x)=-\infty$ for all $n \geq k$. Hence $\Lambda(x)=-\infty$ and $G(x)=\{-\infty\}$, which was to be proved. Next assume that $g_{n}(x)>-\infty$ for every $n \in \mathbb{N}$. Put $c_{n}:=g_{n+1}(x)-g_{n}(x)$ for $n \geq 1$, and $c_{0}:=g_{1}(x)$. Then $c_{n} \leq\left\|g_{1}\right\|_{H^{+}(x)}$ for all $n \in \mathbb{N}$. Now we need the following lemma which is a modification of one used by Walters [46].

LEMMA. If the sequence $\left(c_{n}\right)_{n \in \mathbb{N}}$ in $\mathbb{R} \cup\{-\infty\}$ is bounded from above then the set of all accumulation points of $\left\{n^{-1} \sum_{i=0}^{n-1} c_{i}\right\}_{n \in \mathbb{N}}$ is a connected subset of $\mathbb{R} \cup\{-\infty\}$.

Applying the lemma we get the desired result.

3. Relations between different exponents. Our main results are based on the following inequality.

Proposition 3.1. Let $\mathcal{G}$ be a finite c.s.p., $x \in X$, and $n, m \in \mathbb{N}$. Then

$$
g_{n}(x) \leq m^{-1} \sum_{i=0}^{n-1} g_{m}\left(\theta^{i} x\right)+3 B_{m},
$$

where $B_{m}:=\max \left\{\left\|g_{k}\right\|_{H^{+}(x)}: k=1, \ldots, m\right\}$. 
Pr o of. First suppose that $n \geq m$. We have

$$
\begin{aligned}
g_{n}(x) & =m^{-1} \sum_{i=0}^{m-1} g_{n}(x) \leq m^{-1} \sum_{i=0}^{m-1}\left(g_{n-m+i+1}(x)+g_{m-i-1}\left(\theta^{n-m+i+1} x\right)\right) \\
& \leq m^{-1} \sum_{i=0}^{m-1} g_{n-m+i+1}(x)+B_{m} \\
& \leq m^{-1} \sum_{i=0}^{m-1}\left(g_{n-m+i+1}(x)-g_{i}(x)\right)+2 B_{m},
\end{aligned}
$$

where $g_{0}:=0$. Applying the identity

$$
\sum_{i=0}^{m-1}\left(a_{k+i}-a_{i}\right)=\sum_{i=0}^{k-1}\left(a_{m+i}-a_{i}\right)
$$

we see that

$$
\begin{aligned}
g_{n}(x) & \leq m^{-1} \sum_{i=0}^{n-m}\left(g_{m+i}(x)-g_{i}(x)\right)+2 B_{m} \leq m^{-1} \sum_{i=0}^{n-m} g_{m}\left(\theta^{i} x\right)+2 B_{m} \\
& =m^{-1} \sum_{i=0}^{n-1} g_{m}\left(\theta^{i} x\right)-m^{-1} \sum_{i=n-m+1}^{n-1} g_{m}\left(\theta^{i} x\right)+2 B_{m} \\
& \leq m^{-1} \sum_{i=0}^{n-1} g_{m}\left(\theta^{i} x\right)+3 B_{m},
\end{aligned}
$$

as required.

Now consider the case $n<m$. We have $g_{n}(x) \leq B_{m}$ and

$$
\left|m^{-1} \sum_{i=0}^{n-1} g_{m}\left(\theta^{i} x\right)\right| \leq \frac{n}{m} B_{m} \leq B_{m} .
$$

Hence

$$
g_{n}(x) \leq m^{-1} \sum_{i=0}^{n-1} g_{m}\left(\theta^{i} x\right)+2 B_{m} .
$$

Now we can prove the main result of this section.

THEOREM 3.2 (upper bounds for characteristic exponents). Let $\mathcal{G}$ be a c.s.p., $x \in X$ and $A$ be a non-empty compact invariant subset of $X$. Then

(i) $\bar{\Lambda}(x) \leq \bar{\Omega}(x) \leq \Sigma(x)$,

(ii) $\underline{\Lambda}(x) \leq \underline{\Omega}(x)$,

(iii) $\Sigma(A)=\max \{\underline{\Lambda}(x): x \in A\}$,

(iv) $\Sigma(x)=\max \left\{\underline{\Lambda}(y): y \in H^{+}(x)\right\}$. 
Pr o of. First assume that $\mathcal{G}$ is finite. Let $x \in X$ and $m \in \mathbb{N}$. Applying first Proposition 3.1 and then Proposition 1.7(i) we get

Hence

$$
\begin{aligned}
\limsup _{n \rightarrow+\infty} n^{-1} g_{n}(x) & \leq \limsup _{n \rightarrow+\infty} n^{-1} \sum_{i=0}^{n-1} m^{-1} g_{m}\left(\theta^{i} x\right) \\
& =\max \left\{m^{-1} \int g_{m} d \mu: \mu \in V(x)\right\}
\end{aligned}
$$

$$
\bar{\Lambda}(x) \leq \inf _{m \in \mathbb{N}} \max \left\{m^{-1} \int g_{m} d \mu: \mu \in V(x)\right\},
$$

which proves the first inequality of (i), by Proposition 2.7(iii). Using Lemma 2.6(iv),(v) we can reduce the general case to the finite one. The proof of (ii) is analogous. The second inequality in (i) follows from Proposition 1.5(i).

We now prove (iii). By (i), it is enough to show that $\Sigma(A) \leq \max \{\underline{\Lambda}(x)$ : $x \in A\}$. From Proposition 2.7(vii) it follows that there exists $\nu \in \mathcal{M}^{\Theta}(A)$ such that $\nu$ is ergodic and $\Sigma(A)=\Lambda(\nu)$. Moreover, by Theorem $1.11, \Lambda(\nu)=$ $\underline{\Lambda}(x)$ for some $x \in A$, which completes the proof of (iii). Taking $A=H^{+}(x)$ we obtain (iv).

R e m a r k. The inequalities between characteristic and central exponents stated above correspond to the "easy half" of the proof of the Kingman subadditive ergodic theorem [29]. Note, however, that the estimates used there do not suffice to establish our result.

Now we give some examples to show that a lower characteristic or central exponent need not be equal to the upper one and that the inequalities in Theorem 3.2 may be strict.

Example 3.3. Set $X=\{0,1\}$ and $\theta(0)=\theta(1)=0$. Define $\mathcal{G}:=\left(g_{n}\right)_{n \in \mathbb{N}}$ by $g_{n}(0)=n$ and $g_{n}(1)=\sum_{i=0}^{n-1} \varepsilon_{i}$ for $n \in \mathbb{N}$, where $\varepsilon_{i}=0,1$ for $i \in \mathbb{N}$ and the sequence $n^{-1} \sum_{i=0}^{n-1} \varepsilon_{i}$ is divergent. Then it is easy to show that $\mathcal{G}$ is a c.s.p. and $\underline{\Lambda}(1)<\bar{\Lambda}(1)$. In [22], [23] and [46] we can find examples of c.s.p.'s generated by cocycles for which the strict characteristic exponents do not exist at some points.

EXAMPLE 3.4. Let $\Theta$ be an arbitrary semidynamical system which is minimal and not uniquely ergodic. Then there exist $f \in C(X)$ and $x \in X$ such that the sequence $n^{-1} \sum_{i=0}^{n-1} f\left(\theta^{i} x\right)$ is divergent. If $\mathcal{G}$ is the additive process generated by $f$ then $\underline{\Lambda}(x)=\underline{\Omega}(x)<\bar{\Omega}(x)=\bar{\Lambda}(x)$.

ExAmple 3.5. Let $X, \Theta$ and $\mathcal{G}$ be as in Example 2.9. Then $V(x)=$ $S(x)=\left\{\delta_{x_{0}}\right\}$ for $x \in X$. Hence $\Lambda(x)=-W(x)<0=\Omega(x)=\Sigma(x)$ for $x \neq x_{0}$.

ExAmple 3.6. Let $X=\{0,1\}^{\mathbb{N}}$ and let $\Theta$ be the one-sided shift. Consider the two Bernoulli measures $\mu_{1}=B(1 / 3,2 / 3), \mu_{2}=B(1 / 2,1 / 2)$, and $g \in$ 
$C(X)$ such that $\int g d \mu_{1}<\int g d \mu_{2}$. Take $x_{0} \in X$ with $V\left(x_{0}\right)=\left\{\mu_{1}\right\}$ and $H^{+}\left(x_{0}\right)=X$. Consider the additive process generated by $g$. Then $\Omega\left(x_{0}\right)=$ $\Lambda\left(\mu_{1}\right)=\int g d \mu_{1}$ and $\Sigma\left(x_{0}\right)=\max \left\{\Lambda(\mu): \mu \in \mathcal{M}^{\Theta}(X)\right\} \geq \int g d \mu_{2}$. Hence $\Omega\left(x_{0}\right)<\Sigma\left(x_{0}\right)$.

Definition 3.7. Let $\mathcal{G}:=\left(g_{n}\right)_{n \in \mathbb{N}}$ be a c.s.p. We say that $x \in X$ is normal if $\underline{\Lambda}(x)=\underline{\Omega}(x)$ and $\bar{\Lambda}(x)=\bar{\Omega}(x)$. We call $x \in X$ regular if $\underline{\Lambda}(x)=\bar{\Lambda}(x)$, i.e., the strict characteristic exponent at this point exists.

From the Kingman subadditive ergodic theorem it follows that the set of all normal regular points is of total measure. If a process is additive then each point is normal, by Proposition 1.7(i), but not necessarily regular, as is seen from Example 3.4. In Section 5 we state some sufficient conditions for all points to be normal or regular. One of the simplest conditions which guarantees normality and regularity is the equicontinuity of $\left\{n^{-1} g_{n}\right\}_{n \in \mathbb{N}}$ together with the invariance of the characteristic exponent:

Proposition 3.8. Let $\mathcal{G}:=\left(g_{n}\right)_{n \in \mathbb{N}}$ be a finite c.s.p. Then

(i) if the functions $\left\{n^{-1} g_{n}\right\}_{n \in \mathbb{N}}$ are equicontinuous then $\underline{\Lambda}$ and $\bar{\Lambda}$ are continuous,

(ii) if $\underline{\Lambda}$ is continuous and invariant then $\Lambda(x)=\Omega(x)=\Sigma(x)$ for all $x \in X$,

(iii) if $\bar{\Lambda}$ is continuous and invariant then $\bar{\Lambda}(x)=\bar{\Omega}(x)=\Sigma(x)$ for all $x \in X$.

Proof. Let $x \in X$ and $\varepsilon>0$. Then there exists $\delta>0$ such that $|x-y|<\delta$ implies $\left|n^{-1} g_{n}(x)-n^{-1} g_{n}(y)\right|<\varepsilon$ for every $y \in X$ and $n \in \mathbb{N}$. Hence, for all $y \in X$,

$$
\max (|\underline{\Lambda}(x)-\underline{\Lambda}(y)|,|\bar{\Lambda}(x)-\bar{\Lambda}(y)|) \leq \limsup _{n \rightarrow+\infty}\left|n^{-1} g_{n}(x)-n^{-1} g_{n}(y)\right|<\varepsilon .
$$

If $\underline{\Lambda}$ (resp. $\bar{\Lambda}$ ) is continuous and invariant, then it is constant on $H^{+}(x)$. Now, (ii) and (iii) follow from Theorem 3.2.

Corollary 3.9. Let $\mathcal{G}:=\left(g_{n}\right)_{n \in \mathbb{N}}$ be a finite c.s.p. Suppose that the functions $\left\{n^{-1} g_{n}\right\}_{n \in \mathbb{N}}$ are equicontinuous and $\underline{\Lambda}$ is invariant. Then each $x \in$ $X$ is normal and regular. Moreover, the sequence $\left\{n^{-1} g_{n}\right\}_{n \in \mathbb{N}}$ is uniformly convergent.

Proof. The first assertion follows from Proposition 3.8. As the strict characteristic exponent exists everywhere, the sequence $\left\{n^{-1} g_{n}\right\}_{n \in \mathbb{N}}$ is pointwise convergent. Now, the uniform convergence follows from equicontinuity.

Remarks. Theorem 3.2(iii) was proved independently by Thieullen ([44, Lemma 2.3.5]) in the general case and by Eden ([14, Theorem 3], $[15$, Corollary 3.6]) for smooth semidynamical systems in separable Hilbert 
spaces. Methods developed in the present paper can be used to obtain an affirmative answer to Eden's conjecture concerning relations between the local and global Lyapunov dimension of an attractor ([14, p. 409, Question 1]).

4. Formulae for central, singular and global exponents. In this section we give theorems which enable us to calculate the central and singular exponents at a point from the values of the c.s.p. on the trajectory of that point. We also characterize central and singular exponents in terms of central functions and numbers.

TheOREM 4.1 (formulae for central exponents, I). Let $\mathcal{G}:=\left(g_{n}\right)_{n \in \mathbb{N}}$ be a finite c.s.p. and $x \in X$. Then

(i) $\underline{\Omega}(x)=\inf _{m \in \mathbb{N}} \liminf _{k \rightarrow+\infty}(m k)^{-1} \sum_{i=0}^{k-1} g_{m}\left(\theta^{i} x\right)$,

(2) (ii) $\bar{\Omega}(x)=\inf _{m \in \mathbb{N}} \limsup _{k \rightarrow+\infty}(m k)^{-1} \sum_{i=0}^{k-1} g_{m}\left(\theta^{i} x\right)$,

(iii) in (1) and (2), "inf ${ }_{m \in \mathbb{N}}$ " can be replaced by " $\lim _{m \rightarrow+\infty}$ ".

Proof. The assertions follow immediately from Propositions 1.7(i) and 2.7(ii), (iii).

R e m a r k. The theorem need not be true if $\mathcal{G}$ is not finite. Let $X$ and $\Theta$ be as in Example 3.3. Define $\mathcal{G}:=\left(g_{n}\right)_{n \in \mathbb{N}}$ by $g_{n}(0)=n$ and $g_{n}(1)=-\infty$ for $n \in \mathbb{N}$. Then $\Omega(1)=\Lambda\left(\delta_{0}\right)=1$ but the right-hand sides of $(1)$ and $(2)$ are $-\infty$.

Let $F: \mathbb{N} \rightarrow \mathbb{R}$. We write $\bar{F}$ for $\lim _{\sup _{n \rightarrow+\infty}} n^{-1} \sum_{i=0}^{n-1} F(i)$.

TheOrem 4.2. Let $\mathcal{G}:=\left(g_{n}\right)_{n \in \mathbb{N}}$ be a finite c.s.p. and $x \in X$. Then

$$
\bar{\Omega}(x)=\inf \{\overline{R(\cdot, x)}: R \in \mathcal{R}\} .
$$

Pr o of. Let $x \in X, R \in \mathcal{R}$ and $\varepsilon>0$. Then, applying (2), we obtain for some $D \in \mathbb{R}$,

$$
\begin{aligned}
\bar{\Omega}(x) & \leq \inf _{m \in \mathbb{N}} \limsup _{k \rightarrow+\infty} k^{-1} \sum_{j=0}^{k-1} m^{-1}\left(D+\sum_{i=j}^{m+j-1}(R(i, x)+\varepsilon)\right) \\
& =\inf _{m \in \mathbb{N}}\left(\varepsilon+m^{-1} D+m^{-1} \limsup _{k \rightarrow+\infty} \sum_{i=0}^{m-1}\left(k^{-1} \sum_{j=0}^{k-1} R(i+j, x)\right)\right)
\end{aligned}
$$




$$
\begin{aligned}
& \leq \varepsilon+\inf _{m \in \mathbb{N}} m^{-1} \sum_{i=0}^{m-1}\left(\limsup _{k \rightarrow+\infty} k^{-1} \sum_{j=0}^{k-1} R(i+j, x)\right) \\
& =\varepsilon+\inf _{m \in \mathbb{N}} m^{-1} \sum_{i=0}^{m-1} \overline{R(\cdot, x)}=\varepsilon+\overline{R(\cdot, x)} .
\end{aligned}
$$

Hence $\bar{\Omega}(x) \leq \inf \{\overline{R(\cdot, x)}: R \in \mathcal{R}\}$. To prove the opposite inequality we define $R_{m}: \mathbb{N} \times X \rightarrow \mathbb{R}$ by

$$
R_{m}(k, x):=m^{-1} g_{m}\left(\theta^{k} x\right) \quad \text { for } k, m \in \mathbb{N} \text { and } x \in X .
$$

We show that $R_{m}$ is central. Let $x \in X$ and $\varepsilon>0$. We put $D_{m}:=3 B_{m}$, where $B_{m}$ is as in the assertion of Proposition 3.1. From Proposition 3.1 we get

$$
g_{n}\left(\theta^{k} x\right) \leq m^{-1} \sum_{i=0}^{n-1} g_{m}\left(\theta^{i+k} x\right)+3 B_{m} \leq \sum_{i=k}^{n+k-1} R_{m}(i, x)+D_{m}+\varepsilon,
$$

as required. Applying Theorem 4.1(ii) we conclude that

$$
\begin{aligned}
\inf \{\overline{R(\cdot, x)}: R \in \mathcal{R}\} & \leq \inf \left\{\overline{R_{m}(\cdot, x)}: m \in \mathbb{N}\right\} \\
& =\inf _{m \in \mathbb{N}} \limsup _{k \rightarrow+\infty} k^{-1} \sum_{j=0}^{k-1} R_{m}(j, x) \\
& =\inf _{m \in \mathbb{N}} \limsup _{k \rightarrow+\infty} k^{-1} \sum_{j=0}^{k-1} m^{-1} g_{m}\left(\theta^{j} x\right)=\bar{\Omega}(x) .
\end{aligned}
$$

Theorem 4.3 (formula for the global exponent). Let $\mathcal{G}:=\left(g_{n}\right)_{n \in \mathbb{N}}$ be a c.s.p. and let $A$ be a non-empty compact invariant subset of $X$. Then

$$
\Sigma(A)=\inf _{n \in \mathbb{N}} \max _{A} n^{-1} g_{n}=\lim _{n \rightarrow+\infty} \max _{A} n^{-1} g_{n} .
$$

Proof. First note that the sequence $\left\{\max _{A} g_{n}\right\}_{n \in \mathbb{N}}$ is subadditive. Thus the second equality follows from Lemma 1.6. To prove the first, assume that $\mathcal{G}$ is finite. Set $t:=\inf _{n \in \mathbb{N}} \max _{A} n^{-1} g_{n}$. Then, for each $n \in \mathbb{N}$, there is $x_{n} \in A$ such that $n^{-1} g_{n}\left(x_{n}\right) \geq t$. Now, using Proposition 3.1, and then letting $n \rightarrow$ $+\infty$, we get $t \leq \int m^{-1} g_{m} d \mu$ for each $m \in \mathbb{N}$ and each accumulation point $\mu$ of the sequence $n^{-1} \sum_{i=0}^{n-1} \delta_{\theta^{i} x_{n}}$. Hence $t \leq \Lambda(\mu)$. Thus, by Lemma 1.4, $t \leq \sup \left\{\Lambda(\mu): \mu \in \mathcal{M}^{\Theta}(A)\right\}$. The reverse inequality is evident. This settles the finite case. For arbitrary $\mathcal{G}$, by Lemma 2.6 (vii) we have

$$
\Sigma(A)=\inf _{N \in \mathbb{N}} \Sigma^{(N)}(A)=\inf _{N \in \mathbb{N}} \inf _{n \in \mathbb{N}} \max _{A} n^{-1} g_{n}^{(N)}=\inf _{n \in \mathbb{N}} \max _{A} n^{-1} g_{n}
$$

THEOREM 4.4. Let $\mathcal{G}$ be a c.s.p. and let $A$ be a non-empty compact invariant subset of $X$. Then

$$
\Sigma(A)=\inf \mathcal{R}_{A}
$$


$\operatorname{Pro}$ of. First assume that $\mathcal{G}$ is finite. Let $r \in \mathcal{R}_{A}$ and $\varepsilon>0$. Then there is $D \in \mathbb{R}$ such that

$$
\max _{A} n^{-1} g_{n} \leq n^{-1} D+r+\varepsilon
$$

for all $n \in \mathbb{N}$. Hence $\inf _{n \in \mathbb{N}} \max _{A} n^{-1} g_{n} \leq r$, and by Theorem 4.3, $\Sigma(A) \leq r$. To prove the reverse inequality put $r_{m}:=\max _{A} m^{-1} g_{m}$ for $m \in \mathbb{N}$. We now show that $r_{m}$ is a central number of $A$. Let $x \in X, n \in \mathbb{N}$ and $\varepsilon>0$. By Proposition 3.1, putting $D_{m}:=3 B_{m}$, we get

$$
g_{n}(x) \leq m^{-1} \sum_{i=0}^{n-1} g_{m}\left(\theta^{i} x\right)+3 B_{m} \leq n\left(r_{m}+\varepsilon\right)+D_{m},
$$

which means that $r_{m} \in \mathcal{R}_{A}$. Finally, from Theorem 4.3 we have

$$
\inf \mathcal{R}_{A} \leq \inf _{m \in \mathbb{N}} r_{m}=\Sigma(A),
$$

which was to be shown.

Now we show how to reduce the general case to the finite one. Let $N \in \mathbb{N}$ . We denote by $\mathcal{R}_{A}^{(N)}$ the set of all central numbers of $A$ for the process

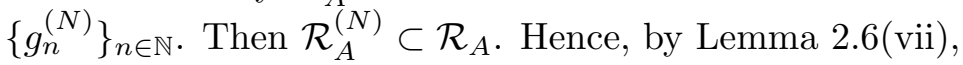

$$
\Sigma(A)=\inf _{N \in \mathbb{N}} \Sigma^{(N)}(A)=\inf _{N \in \mathbb{N}} \inf \mathcal{R}_{A}^{(N)} \geq \inf \mathcal{R}_{A} .
$$

Let now $r \in \mathcal{R}_{A}$. Then clearly $r^{(N)}=\max (r,-N) \in \mathcal{R}_{A}^{(N)}$. Therefore

$$
\Sigma(A)=\inf _{N \in \mathbb{N}} \inf \mathcal{R}_{A}^{(N)} \leq \inf _{N \in \mathbb{N}} \inf \left\{r^{(N)}: r \in \mathcal{R}_{A}\right\}=\inf \mathcal{R}_{A} .
$$

Singular exponents can be calculated in many various ways as the following theorem shows.

THEOREM 4.5 (formulae for singular exponents, I). Let $\mathcal{G}$ be a c.s.p. and $x \in X$. Then

(i) $\Sigma(x)=\inf _{m \in \mathbb{N}} \inf _{k \in \mathbb{N}}(m k)^{-1} \sup _{j \in \mathbb{N}} \sum_{i=0}^{k-1} g_{m}\left(\theta^{i+j} x\right)$,

(ii) $\Sigma(x)=\inf _{m \in \mathbb{N}} \sup _{k \in \mathbb{N}} m^{-1} g_{m}\left(\theta^{k} x\right)$,

(iii) $\Sigma(x)=\limsup _{m, k \rightarrow+\infty} m^{-1} g_{m}\left(\theta^{k} x\right)$,

(iv) in (6), "inf $\operatorname{infN}_{m \in \mathbb{N}}$ " can be replaced by " $\lim _{m \rightarrow+\infty}$ " or " $\inf _{k \in \mathbb{N}}$ " by " $\lim _{k \rightarrow+\infty}$ ", or " $\sup _{j \in \mathbb{N}}$ " by "limsup $\sup _{j \rightarrow+\infty}$ " (also, all three replacements, or any two of them, can be made simultaneously),

(v) in (7), "inf ${ }_{m \in \mathbb{N}}$ " can be replaced by " $\lim _{m \rightarrow+\infty}$ " or " $\sup _{k \in \mathbb{N}}$ " by "lim $\sup _{k \rightarrow+\infty} "$ (or both). 
Pro of. St ep 1. First assume that $\mathcal{G}$ is finite. The assertions (i) and (iv) follow immediately from Propositions 1.7(iii),(iv) and 2.7(iv),(vi). We have

(9) $\limsup _{m \rightarrow+\infty} \lim _{k \rightarrow+\infty} \sup _{j \in \mathbb{N}} k^{-1} \sum_{i=0}^{k-1} A_{m, i+j} \leq \limsup _{m \rightarrow+\infty} \limsup _{k \rightarrow+\infty} A_{m, k}$

$$
\leq \limsup _{m, k \rightarrow+\infty} A_{m, k} \leq \operatorname{limsupsup}_{m \rightarrow+\infty} A_{m, k}
$$

for every double sequence $\left\{A_{m, k}\right\}_{m, k \in \mathbb{N}}$ in $\mathbb{R} \cup\{-\infty\}$. Let $x \in X$. Put $A_{m, k}:=m^{-1} g_{m}\left(\theta^{k} x\right)$ for $m, k \in \mathbb{N}$. Then, by (6), $\Sigma(x)$ is less than or equal to the first term of (9). On the other hand, from Theorem 4.3 it follows that $\Sigma(x)$ is equal to the last term of (9). Hence all the terms in (9) must be equal. This implies (iii) and, by the subadditivity of the sequence $\left\{\lim \sup _{k \rightarrow+\infty} g_{m}\left(\theta^{k} x\right)\right\}_{m \in \mathbb{N}}$, also (ii) and (v). Thus the proof of the finite case is complete.

S tep 2. Let $\mathcal{G}$ be an arbitrary c.s.p. and $x \in X$. It is enough to prove that $\Sigma(x)$ does not exceed the first term of (9), as the other arguments used in step 1 work also in the general case. Let $f: X \rightarrow \mathbb{R} \cup\{-\infty\}$ be continuous and let $\mu \in S(x)$. Then

$$
\int f d \mu=\inf _{N \in \mathbb{N}} \int f^{(N)} d \mu \leq \inf _{N \in \mathbb{N}} \limsup _{j \rightarrow+\infty} f^{(N)}\left(\theta^{j} x\right)=\limsup _{j \rightarrow+\infty} f\left(\theta^{j} x\right) .
$$

Applying this to $f:=k^{-1} \sum_{i=0}^{k-1} m^{-1} g_{m}\left(\theta^{i} x\right)$, for $k, m \in \mathbb{N}$, we get the desired result.

TheOrem 4.6. Let $\mathcal{G}$ be a c.s.p. and $x \in X$. Then

$$
\Sigma(x)=\inf \left\{\overline{R(\cdot, x)}: R \in \mathcal{R}_{0}\right\} .
$$

Proof. The assertion follows immediately from Theorem 4.4.

Remarks. (1) The results obtained in this section allow us to identify the notions of exponents that come from the theory of linear differential equations with those introduced in the present paper and hence justify our terminology. In [8, pp.116-117] the authors define upper central and singular exponents for a non-autonomous linear differential equation in $\mathbb{R}^{d}$ by the formulae (3) and (10) (see also [45]). They show that (2) and (7) hold in this case and prove an analogue of Theorem 3.2(i).

(2) For a c.s.p. generated by a cocycle the singular exponent can also be defined as the top element of the continuous spectrum of the linear skew product system associated with the cocycle. From [24, Theorem 2.3] it follows that this definition coincides with our general definition. In [24] it is also shown that (8) holds in that case.

(3) Global exponents were introduced by means of formula (4) in the paper of Constantin and Foiaş on attractors for the Navier-Stokes equa- 
tion [10]. In [43] Temam generalized this, considering characteristic and global exponents for arbitrary smooth infinite-dimensional dynamical systems. He called them Lyapunov pointwise exponents and Lyapunov uniform (or global) exponents respectively.

(4) In the proof of Theorem 4.3 we use ideas similar to those of Ledrappier in the proof of a formula for the dilating dimension of the Lyapunov spectrum ([31, Proposition 3]).

5. Conjugate processes. One of the main features which make subadditive ergodic theory different from its additive counterpart is asymmetry. In general, characteristic exponents are only subinvariant, we only have inequality between characteristic and central exponents and only some points are regular, in contrast to the additive case. In this section we propose a new method which allows us to improve these results, at least in some cases. We introduce the notion of conjugate processes, which generalizes the concept of an exact dominant of a measurable superadditive processes used in the $L^{1}$ case [30, p. 147].

In this section we consider finite c.s.p.'s only.

Definition 5.1. Suppose that $\mathcal{G}:=\left(g_{n}\right)_{n \in \mathbb{N}}$ and $\mathcal{H}:=\left(h_{n}\right)_{n \in \mathbb{N}}$ are finite c.s.p.'s. We say that $\mathcal{H}$ is conjugate to $\mathcal{G}$ (or $\mathcal{G}$ and $\mathcal{H}$ are conjugate) if

$$
h_{n}+g_{n} \geq 0 \quad \text { for each } n \in \mathbb{N} \text {. }
$$

Central and singular exponents of conjugate processes are related in the following way.

Proposition 5.2. Let $\mathcal{G}:=\left(g_{n}\right)_{n \in \mathbb{N}}$ and $\mathcal{H}:=\left(h_{n}\right)_{n \in \mathbb{N}}$ be conjugate finite c.s.p.'s. Then

(i) $0 \leq \Lambda_{\mathcal{G}}(\mu)+\Lambda_{\mathcal{H}}(\mu)$ for $\mu \in \mathcal{M}^{\Theta}(X)$,

(ii) $0 \leq \bar{\Omega}_{\mathcal{G}}(x)+\bar{\Omega}_{\mathcal{H}}(x) \leq \Sigma_{\mathcal{G}}(x)+\Sigma_{\mathcal{H}}(x) \leq \Sigma_{\mathcal{G}}(X)+\Sigma_{\mathcal{H}}(X)$ for $x \in X$.

Proof. Let $\mu \in \mathcal{M}^{\Theta}(X)$. We have

$$
\begin{aligned}
0 & \leq \lim _{n \rightarrow+\infty} \int n^{-1}\left(g_{n}+h_{n}\right) d \mu \\
& =\lim _{n \rightarrow+\infty} \int n^{-1} g_{n} d \mu+\lim _{n \rightarrow+\infty} \int n^{-1} h_{n} d \mu=\Lambda_{\mathcal{G}}(\mu)+\Lambda_{\mathcal{H}}(\mu),
\end{aligned}
$$

which proves (i). The assertion (ii) follows immediately from (i).

Definition 5.3. Let $\mathcal{G}:=\left(g_{n}\right)_{n \in \mathbb{N}}$ be a finite c.s.p. Suppose that $\mathcal{H}:=$ $\left(h_{n}\right)_{n \in \mathbb{N}}$ is a finite c.s.p. conjugate to $\mathcal{G}$. We say that $\mathcal{H}$ satisfies condition

$(\alpha)$ if $g_{k} \circ \theta^{n} \leq g_{n+k}+h_{n}$ for all $n, k \in \mathbb{N}$,

( $\beta) \quad$ if $g_{n} \leq g_{n+k}+h_{k} \circ \theta^{n}$ for all $n, k \in \mathbb{N}$,

$(\gamma)$ if $\Sigma_{\mathcal{G}}(X)+\Sigma_{\mathcal{H}}(X)=0$

$(\delta)$ if $\Sigma_{\mathcal{G}}(x)+\Sigma_{\mathcal{H}}(x)=0$ for all $x \in X$, 
$(\varepsilon) \quad$ if $\bar{\Omega}_{\mathcal{G}}(x)+\bar{\Omega}_{\mathcal{H}}(x)=0$ for all $x \in X$,

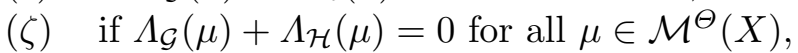

( $\eta)$ if the sequence $\left\{\left(g_{n}+h_{n}\right) / n\right\}_{n \in \mathbb{N}}$ converges to 0 , uniformly on each non-empty compact invariant set, as $n \rightarrow+\infty$.

Proposition 5.4. In the above situation the following implications hold:

$$
(\gamma) \Rightarrow(\delta) \Rightarrow(\varepsilon) \Rightarrow(\zeta) \Leftrightarrow(\eta) .
$$

P r o of. The first two implications follow directly from Proposition 5.2(ii). We show that $(\zeta)$ implies $(\eta)$. Let $A$ be a non-empty compact invariant set and $\mu \in \mathcal{M}^{\Theta}(A)$. We have $\Lambda_{\mathcal{G}+\mathcal{H}}(\mu)=\Lambda_{\mathcal{G}}(\mu)+\Lambda_{\mathcal{H}}(\mu)=0$. Hence $\Sigma_{\mathcal{G}+\mathcal{H}}(A)=0$. Then, by Theorem $4.3, \lim _{n \rightarrow+\infty} n^{-1} \max \left\{g_{n}(x)+h_{n}(x)\right.$ : $x \in A\}=0$, as required.

By Proposition 1.12, to prove that $(\eta)$ or $(\varepsilon)$ implies $(\zeta)$ it is enough to show that $(\zeta)$ is satisfied for all ergodic measures. Let $\nu$ be one. By the Birkhoff ergodic theorem, there exists $x_{0}$ such that $V\left(x_{0}\right)=\{\nu\}$. Consequently, $\operatorname{supp} \nu \subset H^{+}\left(x_{0}\right)$. First, assume that $(\varepsilon)$ holds. Then $\Lambda_{\mathcal{G}}(\nu)+$ $\Lambda_{\mathcal{H}}(\nu)=\bar{\Omega}_{\mathcal{G}}\left(x_{0}\right)+\bar{\Omega}_{\mathcal{H}}\left(x_{0}\right)=0$. On the other hand, if $(\eta)$ is satisfied, then Proposition 5.2(i) yields

$$
\begin{aligned}
0 & \leq \Lambda_{\mathcal{G}}(\nu)+\Lambda_{\mathcal{H}}(\nu)=\lim _{n \rightarrow+\infty} \int n^{-1}\left(g_{n}+h_{n}\right) d \nu \\
& \leq \lim _{n \rightarrow+\infty} n^{-1}\left\|g_{n}+h_{n}\right\|_{H^{+}\left(x_{0}\right)}=0 .
\end{aligned}
$$

None of the first three implications of Proposition 5.4 can be reversed. Counterexamples can be constructed even in the additive case.

Example $5.5(\delta \nRightarrow \gamma)$. Put $X=\{0,1\}, \theta(0)=0$ and $\theta(1)=1$. Let $\mathcal{G}$ and $\mathcal{H}$ be the additive processes generated by $g$ and $h$ respectively, where $g(0)=-1, g(1)=0, h(0)=1$ and $h(1)=0$. Then $\Sigma_{\mathcal{G}}(0)+\Sigma_{\mathcal{H}}(0)=$ $\Sigma_{\mathcal{G}}(1)+\Sigma_{\mathcal{H}}(1)=0$ but $\Sigma_{\mathcal{G}}(X)+\Sigma_{\mathcal{H}}(X)=1$.

EXAmple 5.6 $(\varepsilon \nRightarrow \delta)$. Let $Y=\{0,1\}^{\mathbb{N}}$ and $\Theta$ be the one-sided shift. Let $x_{0} \in Y$ be the sequence consisting of blocs of $n$ ones and $2^{n}$ zeros occurring alternately. Consider the subshift generated by $x_{0}$, i.e., $X=H^{+}\left(x_{0}\right)$. Let $\mathcal{G}$ and $\mathcal{H}$ be the additive processes generated by $g$ and $h$ respectively, where $g(x)=x(0)$ for $x \in X$ and $h=-g$. For $n \in \mathbb{N}$, denote by $\alpha_{n}$ the sequence of $n$ zeros followed by ones and by $\beta_{n}$ the sequence of $n$ ones followed by zeros. Then $L^{+}\left(x_{0}\right)=\left\{\alpha_{n}: n \in \mathbb{N}\right\} \cup\left\{\beta_{n}: n \in \mathbb{N}\right\}, X=\gamma^{+}\left(x_{0}\right) \cup L^{+}\left(x_{0}\right)$ and $\mathcal{M}^{\Theta}(X)=\left\{\delta_{\alpha_{0}}, \delta_{\beta_{0}}\right\}$. Moreover, for each $k \in \mathbb{N}$, we have $V\left(\theta^{k} x_{0}\right)=$ $V\left(\beta_{k}\right)=S\left(\beta_{k}\right)=\left\{\delta_{\beta_{0}}\right\}, V\left(\alpha_{k}\right)=S\left(\alpha_{k}\right)=\left\{\delta_{\alpha_{0}}\right\}$ and $S\left(\theta^{k} x_{0}\right)=\mathcal{M}^{\Theta}(X)$. Hence $\Omega_{\mathcal{G}}(x)+\Omega_{\mathcal{H}}(x)=0$ for each $x \in X$, but $\Sigma_{\mathcal{G}}\left(x_{0}\right)+\Sigma_{\mathcal{H}}\left(x_{0}\right)=1$.

ExAmPle $5.7(\zeta \nRightarrow \varepsilon)$. Let $X, \Theta, \mu_{1}, \mu_{2}$ and $g$ be as in Example 3.6. Take $x_{0} \in X$ such that $\mu_{1}, \mu_{2} \in V\left(x_{0}\right)$. Consider the additive processes $\mathcal{G}$ and 
$\mathcal{H}$ generated by $g$ and $h=-g$ respectively. Clearly $\Lambda_{\mathcal{G}}(\mu)+\Lambda_{\mathcal{H}}(\mu)=0$ for each $\mu \in \mathcal{M}^{\Theta}(X)$. However, $\bar{\Omega}_{\mathcal{G}}\left(x_{0}\right)+\bar{\Omega}_{\mathcal{H}}\left(x_{0}\right) \geq \int g d \mu_{2}-\int g d \mu_{1}>0$.

We now describe some general methods of constructing conjugate processes for c.s.p.'s generated by cocycles.

Proposition 5.8. Let $\Phi: X \times \mathbb{N} \rightarrow \mathrm{Gl}(d)$ be a cocycle over $\Theta$ and let $\mathcal{G}$ be the c.s.p. generated by $\Phi$. Then

$$
h_{n}(x):=\ln \left\|\Phi^{-1}(x, n)\right\| \quad \text { for } x \in X \text { and } n \in \mathbb{N}
$$

defines a c.s.p. $\mathcal{H}$ conjugate to $\mathcal{G}$ and satisfying conditions $(\alpha)$ and $(\beta)$.

If $\mu \in \mathcal{M}^{\Theta}(X)$, we write $\Lambda^{*}(\mu)$ for $-\Lambda_{\mathcal{H}}(\mu)$.

Pr o of. Let $x \in X$ and $n, k \in \mathbb{N}$. The asserted properties of $\mathcal{H}$ follow from the identities $\Phi^{-1}(x, n+k)=\Phi^{-1}(x, n) \circ \Phi^{-1}\left(\theta^{n} x, k\right), I=\Phi^{-1}(x, n) \circ \Phi(x, n)$, $\Phi\left(\theta^{n} x, k\right)=\Phi(x, n+k) \circ \Phi^{-1}(x, n), \Phi(x, n)=\Phi^{-1}\left(\theta^{n} x, k\right) \circ \Phi(x, n+k)$ and the inequality $\|A \circ B\| \leq\|A\| \cdot\|B\|$.

Proposition 5.9. Let $\mathcal{G}:=\left(g_{n}\right)_{n \in \mathbb{N}}$ be a finite c.s.p. which satisfies the following condition:

(*) $\quad$ there exists $c \in \mathbb{R}^{+}$such that $g_{k}\left(\theta^{n} x\right)+g_{n}(x) \leq g_{n+k}(x)+c$ for all $x \in X, n, k \in \mathbb{N}$.

Then $h_{n}:=c-g_{n}$ for $n \in \mathbb{N}$ defines a c.s.p. $\mathcal{H}$ conjugate to $\mathcal{G}$ and satisfying conditions $(\alpha),(\beta),(\zeta)$ and $(\eta)$.

Proof. From $(*)$ we see immediately that $\mathcal{H}$ is a c.s.p. and satisfies $(\alpha)$ and $(\beta)$. Moreover, $0 \leq h_{n}(x)+g_{n}(x)=c$ for all $x \in X$ and $n \in \mathbb{N}$. Therefore $\mathcal{H}$ is conjugate to $\mathcal{G}$ and satisfies $(\eta)$, which is equivalent to $(\zeta)$.

Proposition 5.10. Suppose that $X$ is compact. Let $\Phi: X \times \mathbb{N} \rightarrow M(d)$ be a cocycle over $\Theta$ such that, for each $x \in X$, the matrix $\Phi(x, 1)$ has strictly positive entries. Then the c.s.p. generated by $\Phi$ satisfies condition (*).

Proof. In the proof we shall use the following lemma of Walters.

Lemma [46, Lemma 2.3]. Let $A^{(1)}, \ldots, A^{(k)}, B^{(1)}, \ldots, B^{(n)}$ be $d \times d m a$ trices with strictly positive entries. Then $\left\|A^{(k)} \circ \ldots \circ A^{(1)}\right\| \cdot\left\|B^{(1)} \circ \ldots \circ B^{(n)}\right\| \leq C\left\|A^{(k)} \circ \ldots \circ A^{(1)} \circ B^{(1)} \circ \ldots \circ B^{(n)}\right\|$, where $C:=d \max \left\{B_{i j}^{(1)} / B_{h j}^{(1)}: i, j, h=1, \ldots, d\right\}$ and $\|A\|:=\sum_{i, j=1}^{d}\left|A_{i j}\right|$.

Let $x \in X$ and $n, k \in \mathbb{N}$. We apply the above lemma to $A^{(i)}:=$ $\Phi\left(\theta^{n+i-1} x, 1\right)$ for $i=1, \ldots, k$ and $B^{(j)}:=\Phi\left(\theta^{n-j} x, 1\right)$ for $j=1, \ldots, n$. As all norms in $M(d)$ are equivalent, there exists $D \in \mathbb{R}^{+}$(independent of $x, n, k)$ such that

$$
\|\Phi(x, n)\| \cdot\left\|\Phi\left(\theta^{n} x, k\right)\right\| \leq C\|\Phi(x, n+k)\|,
$$


where

$$
C:=\max \left\{\Phi_{i j}\left(\theta^{n-1} x, 1\right) / \Phi_{h j}\left(\theta^{n-1} x, 1\right): i, j, h=1, \ldots, d\right\} \cdot D d .
$$

Hence $g_{n}(x)+g_{k}\left(\theta^{n} x\right) \leq g_{n+k}(x)+c$, where

$$
\begin{aligned}
c:= & \ln \max \left\{\Phi_{i j}(y, 1): y \in X, i, j=1, \ldots, d\right\} \\
& -\ln \min \left\{\Phi_{i j}(y, 1): y \in X, i, j=1, \ldots, d\right\}+\ln D d .
\end{aligned}
$$

Using conjugate processes we get lower bounds for characteristic exponents.

THEOREM 5.11 (lower bounds for characteristic exponents). Suppose that $\mathcal{G}:=\left(g_{n}\right)_{n \in \mathbb{N}}$ is a finite c.s.p., $\mathcal{H}:=\left(h_{n}\right)_{n \in \mathbb{N}}$ is a finite c.s.p. conjugate to $\mathcal{G}$ and $x \in X$. Then

(i) $-\underline{\Omega}_{\mathcal{H}}(x) \leq \bar{\Lambda}_{\mathcal{G}}(x)$,

(ii) $-\bar{\Omega}_{\mathcal{H}}(x) \leq \underline{\Lambda}_{\mathcal{G}}(x)$.

Proof. Note that

$$
\bar{\Lambda}_{\mathcal{G}}(x)=\limsup _{n \rightarrow+\infty} n^{-1} g_{n}(x) \geq-\liminf _{n \rightarrow+\infty} n^{-1} h_{n}(x)=-\underline{\Lambda}_{\mathcal{H}}(x) .
$$

Applying Theorem 3.2(i) to $\mathcal{H}$ we obtain (i). The proof of (ii) is analogous.

CoROLlary 5.12. Let $\mathcal{G}:=\left(g_{n}\right)_{n \in \mathbb{N}}$ be a finite c.s.p. If there exists a finite c.s.p. conjugate to $\mathcal{G}$, then all lower and upper characteristic exponents are finite.

Pro of. Suppose that $\mathcal{H}:=\left(h_{n}\right)_{n \in \mathbb{N}}$ is a finite c.s.p. conjugate to $\mathcal{G}$ and $x \in X$. Then, by Theorem 5.11, $\underline{\Lambda}_{\mathcal{G}}(x) \geq-\bar{\Omega}_{\mathcal{H}}(x)$. Hence, by Theorems $3.2(\mathrm{i})$ and $4.5(\mathrm{ii}), \underline{\Lambda}_{\mathcal{G}}(x) \geq-\Sigma_{\mathcal{H}}(x) \geq-\left\|h_{1}\right\|_{H^{+}(x)}>-\infty$. Finally, Proposition 2.9 gives $\bar{\Lambda}_{\mathcal{G}}(x)<+\infty$.

Corollary 5.13. Let $\Phi: X \times \mathbb{N} \rightarrow \operatorname{Gl}(d)$ be a cocycle over $\Theta, \mathcal{G}$ be the c.s.p. generated by $\Phi$, and $x \in X$. Then

(i) $\sup \left\{\Lambda^{*}(\mu): \mu \in V(x)\right\} \leq \bar{\Lambda}(x)$,

(ii) $\inf \left\{\Lambda^{*}(\mu): \mu \in V(x)\right\} \leq \underline{\Lambda}(x)$

$\left(\Lambda^{*}\right.$ was defined after Proposition 5.8).

Proof. The corollary is an immediate consequence of Proposition 5.8 and Theorem 5.11.

Remark. It is well known that $\Lambda^{*}(\mu)$ is the lowest element of the Lyapunov spectrum of $\mu$. Using this fact and applying the so-called wedge product flows [24] we can obtain results on multidimensional characteristic exponents of a cocycle $\Phi$. 
THEOREM 5.14 (invariance of characteristic exponents). Let $\mathcal{G}:=$ $\left(g_{n}\right)_{n \in \mathbb{N}}$ be a finite c.s.p. If there exists a finite c.s.p. conjugate to $\mathcal{G}$ and satisfying condition $(\alpha)$, then the characteristic exponents are invariant, i.e.,

(i) $\underline{\Lambda}(x)=\underline{\Lambda}\left(\theta^{n} x\right), \bar{\Lambda}(x)=\bar{\Lambda}\left(\theta^{n} x\right)$ and

(ii) $G(x)=G\left(\theta^{n} x\right)$

for all $x \in X$ and $n \in \mathbb{N}$.

Pr o of. Suppose that $\mathcal{H}:=\left(h_{n}\right)_{n \in \mathbb{N}}$ is a finite c.s.p. conjugate to $\mathcal{G}$ and satisfying $(\alpha)$. We have

$$
\begin{aligned}
\underline{\Lambda}(x) & =\liminf _{k \rightarrow+\infty} k^{-1} g_{k}(x)=\liminf _{k \rightarrow+\infty} k^{-1} g_{n+k}(x) \\
& \geq \liminf _{k \rightarrow+\infty}\left(k^{-1} g_{k}\left(\theta^{n} x\right)-k^{-1} h_{n}(x)\right)=\underline{\Lambda}\left(\theta^{n} x\right) .
\end{aligned}
$$

The proof of $\bar{\Lambda}(x) \geq \bar{\Lambda}\left(\theta^{n} x\right)$ is analogous. Now (i) follows from Theorem 2.8(i). The assertion (ii) results from (i) and Proposition 2.10.

Remarks. (1) By Proposition 5.8 the above theorem applies in particular to c.s.p.'s generated by cocycles with values in $\mathrm{Gl}(d)$.

(2) The existence of a finite conjugate c.s.p. satisfying $(\alpha)$ is not necessary for the invariance of characteristic exponents. This can be seen from the following example. Let $X$ and $\Theta$ be as in Example 3.3. Put $g_{n}(0)=0$ and $g_{n}(1)=-n^{1 / 2}$ for $n \in \mathbb{N}$. Then $\mathcal{G}:=\left(g_{n}\right)_{n \in \mathbb{N}}$ is a c.s.p. with $\Lambda(0)=\Lambda(1)=0$ but $g_{k}\left(\theta^{n} 1\right)-g_{n+k}(1)=(n+k)^{1 / 2} \rightarrow+\infty(n \rightarrow+\infty)$ for each $k \in \mathbb{N}$. Consequently, there is no finite c.s.p. conjugate to $\mathcal{G}$ and satisfying $(\alpha)$.

We now show how the existence of a conjugate c.s.p. satisfying an appropriate condition implies normality or regularity.

TheOREM 5.15. Let $\mathcal{G}:=\left(g_{n}\right)_{n \in \mathbb{N}}$ be a c.s.p. and $\mathcal{H}:=\left(h_{n}\right)_{n \in \mathbb{N}}$ be a finite c.s.p. conjugate to $\mathcal{G}$.

(i) If $\mathcal{H}$ satisfies $(\zeta)$ or $(\eta)$, then $\underline{\Lambda}(x)=\underline{\Omega}(x)$ and $\bar{\Lambda}(x)=\bar{\Omega}(x)$ for each $x \in X$, i.e., each point is normal.

(ii) If $\mathcal{H}$ satisfies $(\varepsilon)$, then $\Lambda(x)=\Omega(x)$ for each $x \in X$, i.e., each point is normal and regular.

(iii) If $\mathcal{H}$ satisfies $(\delta)$, then $\Lambda(x)=\Omega(x)=\Sigma(x)$ for each $x \in X$.

(iv) If $\mathcal{H}$ satisfies $(\gamma)$, then $\Lambda(x)=\Omega(x)=\Sigma(x)=\Sigma(X)$ for each $x \in X$.

Proof. In the proof we use Proposition 5.4.

(i) We have

$$
\bar{\Omega}_{\mathcal{H}}(x)=\sup \left\{\Lambda_{\mathcal{H}}(\mu): \mu \in V(x)\right\}=-\inf \left\{\Lambda_{\mathcal{G}}(\mu): \mu \in V(x)\right\}=-\underline{\Omega}_{\mathcal{G}}(x) .
$$

Analogously $\underline{\Omega}_{\mathcal{H}}(x)=-\bar{\Omega}_{\mathcal{G}}(x)$. Hence, applying Theorems 3.2(i), (ii) and $5.11(\mathrm{i})$, (ii) we get

$$
\underline{\Omega}_{\mathcal{G}}(x)=-\bar{\Omega}_{\mathcal{H}}(x) \underline{\Lambda}_{\mathcal{G}}(x) \leq \underline{\Omega}_{\mathcal{G}}(x)
$$


and

$$
\bar{\Omega}_{\mathcal{G}}(x)=-\underline{\Omega}_{\mathcal{H}}(x) \leq \bar{\Lambda}_{\mathcal{G}}(x) \leq \bar{\Omega}_{\mathcal{G}}(x),
$$

which proves (i).

(ii) Using the same arguments as in (i) we obtain $\underline{\Omega}_{\mathcal{G}}(x)=-\bar{\Omega}_{\mathcal{H}}(x)=$ $\bar{\Omega}_{\mathcal{G}}(x)$. Now (ii) results from (i).

(iii) By Theorem 3.2(i) we have

$$
\Sigma_{\mathcal{G}}(x)=-\Sigma_{\mathcal{H}}(x) \leq-\Omega_{\mathcal{H}}(x)=\Omega_{\mathcal{G}}(x) \leq \Sigma_{\mathcal{G}}(x) .
$$

Now apply (ii).

(iv) Clearly $\Sigma_{\mathcal{G}}(X)=-\Sigma_{\mathcal{H}}(X) \leq-\Sigma_{\mathcal{H}}(x)=\Sigma_{\mathcal{G}}(x)=\Sigma_{\mathcal{G}}(X)$ and so (iv) results from (iii).

The following corollary results immediately from the above theorem and Propositions 5.9 and 5.10.

Corollary 5.16. Suppose that $X$ is compact and $\Phi: X \times \mathbb{N} \rightarrow M(d)$ is a cocycle over $\Theta$ such that, for each $x \in X$, the matrix $\Phi(x, 1)$ has strictly positive entries. Then each point is normal with respect to the c.s.p. generated by $\Phi$.

To end this section, we show how conjugate processes can be used to obtain some formulae for central and singular exponents. Let $x \in X$ and $m \in \mathbb{N}$. Recall that $V_{m}(x)$ denotes the set of all accumulation points of the sequence $\left\{n^{-1} \sum_{i=0}^{n-1} \delta_{\theta^{m i} x}\right\}_{n \in \mathbb{N}}$ and $S_{m}(x)$ is the set of all $\theta^{m}$-invariant measures with supports contained in $\operatorname{cl}\left\{\theta^{m i} x: i \in \mathbb{N}\right\}$.

Theorem 5.17 (formulae for central and singular exponents, II). Let $\mathcal{G}:=\left(g_{n}\right)_{n \in \mathbb{N}}$ be a finite c.s.p. and $m \in \mathbb{N}$. Suppose that there exists a finite c.s.p. conjugate to $\mathcal{G}$ and satisfying condition $(\alpha)$. Then

(i) in Proposition 2.7(i)-(iv),(vi), $V(x)$ can be replaced by $V_{m}(x)$, and $S(x)$ by $S_{m}(x)$,

(ii) in Theorem 4.1(ii),(iii), $\theta$ can be replaced by $\theta^{m}$,

(iii) in Theorem 4.5, $\theta$ can be replaced by $\theta^{m}$.

Proof. Let $\mathcal{H}:=\left(h_{n}\right)_{n \in \mathbb{N}}$ be a finite c.s.p. conjugate to $\mathcal{G}$ and satisfying $(\alpha)$.

By Proposition 1.7(i),(iii),(iv) the assertion (i) follows from (ii) and (iii).

Now suppose that $k \in \mathbb{N}$ and $R: \mathbb{N} \times X \rightarrow \mathbb{R}$ is a central function for $\Theta$. Then, for each $\varepsilon>0$ and $x \in X$, there exists $D \in \mathbb{R}$ such that

$$
g_{m}\left(\theta^{i m} x\right) \leq D+\sum_{j=i m}^{i m+m-1}(R(j, x)+\varepsilon) \quad \text { for } i, m \in \mathbb{N} .
$$


Summing over $i=0, \ldots, k-1$ and dividing both sides by $m k$ we get

Hence

$$
(m k)^{-1} \sum_{i=0}^{k-1} g_{m}\left(\theta^{i m} x\right) \leq m^{-1} D+\varepsilon+(m k)^{-1} \sum_{j=0}^{m k-1} R(j, x) .
$$

$$
\limsup _{m \rightarrow+\infty} m^{-1}\left(\limsup _{k \rightarrow+\infty} k^{-1} \sum_{i=0}^{k-1} g_{m}\left(\theta^{i m} x\right)\right) \leq \overline{R(\cdot, x)}+\varepsilon .
$$

As $\varepsilon$ was arbitrary, using Theorem 4.2 , we obtain

$$
\limsup _{m \rightarrow+\infty} m^{-1}\left(\limsup _{k \rightarrow+\infty} k^{-1} \sum_{i=0}^{k-1} g_{m}\left(\theta^{i m} x\right)\right) \leq \bar{\Omega}(x) .
$$

We denote the integer part of $a \in \mathbb{R}$ by $[a]$. Define $R_{m}: \mathbb{N} \times X \rightarrow \mathbb{R}$, for $m \in \mathbb{N}$, by

$$
R_{m}(p, x):=m^{-1} g_{m}\left(\theta^{[p / m] \cdot m} x\right) \quad \text { for } x \in X \text { and } p \in \mathbb{N} .
$$

We now prove that $R_{m}$ is central. Fix $x \in X$ and set $B_{m}:=$ $\max _{k=1, \ldots, m}\left\|g_{k}\right\|_{H^{+}(x)}$ and $C_{m}:=\max _{k=1, \ldots, m}\left\|h_{k}\right\|_{H^{+}(x)}$. Let $n, u \in \mathbb{N}$. Put $k:=[n / m]$ and $j:=[u / m]$. Then

$$
g_{n}\left(\theta^{u} x\right) \leq g_{k m}\left(\theta^{u} x\right)+g_{n-k m}\left(\theta^{u+k m} x\right)
$$

Moreover,

$$
\begin{aligned}
g_{k m}\left(\theta^{u} x\right) & \leq g_{k m+u-j m}\left(\theta^{j m} x\right)+h_{u-j m}\left(\theta^{j m} x\right) \\
& \leq g_{k m}\left(\theta^{j m} x\right)+g_{u-j m}\left(\theta^{j m+k m} x\right)+h_{u-j m}\left(\theta^{j m} x\right) \\
& \leq \sum_{i=j}^{k+j-1} g_{m}\left(\theta^{i m} x\right)+B_{m}+C_{m} .
\end{aligned}
$$

From (12) and (13) we obtain

$$
g_{n}\left(\theta^{u} x\right) \leq \sum_{i=j}^{k+j-1} g_{m}\left(\theta^{i m} x\right)+2 B_{m}+C_{m} .
$$

Finally, we get

$$
\begin{aligned}
g_{n}\left(\theta^{u} x\right) \leq & 2 B_{m}+C_{m}+\sum_{p=j m}^{(k+j) m-1} R_{m}(p, x) \\
= & 2 B_{m}+C_{m}+\sum_{p=u}^{n+u-1} R_{m}(p, x)+\sum_{p=j m}^{u-1} R_{m}(p, x) \\
& -\sum_{p=(k+j) m}^{n+u-1} R_{m}(p, x)
\end{aligned}
$$




$$
\leq 4 B_{m}+C_{m}+\sum_{p=u}^{n+u-1} R_{m}(p, x)
$$

as required.

Now fix $n \in \mathbb{N}$. Then

$$
\begin{aligned}
n^{-1} \sum_{p=0}^{n-1} R_{m}(p, x)= & n^{-1} \sum_{i=0}^{[n / m]-1} g_{m}\left(\theta^{i m} x\right) \\
& +(n m)^{-1} \sum_{p=[n / m] m}^{n-1} g_{m}\left(\theta^{[(n-1) / m] m} x\right) \\
\leq & \frac{[n / m]}{n / m} \cdot \frac{1}{[n / m]} \sum_{i=0}^{[n / m]-1} m^{-1} g_{m}\left(\theta^{i m} x\right)+n^{-1} B_{m} .
\end{aligned}
$$

Hence

$$
\overline{R_{m}(\cdot, x)} \leq \limsup _{k \rightarrow+\infty} k^{-1} \sum_{i=0}^{k-1} m^{-1} g_{m}\left(\theta^{i m} x\right) .
$$

Therefore, applying Theorem 4.2, we have

$$
\begin{aligned}
\bar{\Omega}(x) & \leq \inf _{m \in \mathbb{N}} \limsup _{k \rightarrow+\infty} k^{-1} \sum_{i=0}^{k-1} m^{-1} g_{m}\left(\theta^{i m} x\right) \\
& \leq \liminf _{m \rightarrow+\infty} m^{-1}\left\{\limsup _{k \rightarrow+\infty} k^{-1} \sum_{i=0}^{k-1} g_{m}\left(\theta^{i m} x\right)\right\} .
\end{aligned}
$$

From (11) and (15) the assertion (ii) follows.

To show (iii) we use the estimate (9) from the proof of Theorem 4.5. It is enough to show that

$$
\Sigma(x) \leq \inf _{m \in \mathbb{N}} \lim _{k \rightarrow+\infty} k^{-1} \sup _{j \in \mathbb{N}} \sum_{i=j}^{k+j-1} m^{-1} g_{m}\left(\theta^{i m} x\right)
$$

and

$$
\limsup _{m \rightarrow+\infty} \sup _{k \in \mathbb{N}} m^{-1} g_{m}\left(\theta^{k m} x\right) \leq \Sigma(x)
$$

for $x \in X$.

Fix $m \in \mathbb{N}$. Put $k_{n}:=[n / m]$ and $j_{u}:=[u / m]$. Then, using (14), we have

$$
g_{n}\left(\theta^{u} x\right) \leq \sum_{i=j_{u}}^{k_{n}+j_{u}-1} g_{m}\left(\theta^{i m} x\right)+2 B_{m}+C_{m} .
$$


Hence

$$
\frac{n}{m k_{n}} \sup _{u \in \mathbb{N}} n^{-1} g_{n}\left(\theta^{u} x\right) \leq\left(k_{n}\right)^{-1} \sup _{j \in \mathbb{N}} \sum_{i=j}^{k_{n}+j-1} m^{-1} g_{m}\left(\theta^{i m} x\right)+\frac{2 B_{m}+C_{m}}{m k_{n}} .
$$

Taking the upper limit as $n \rightarrow+\infty$ we get

$$
\Sigma(x)=\limsup _{n \rightarrow+\infty} \sup _{u \in \mathbb{N}} n^{-1} g_{n}\left(\theta^{u} x\right) \leq \lim _{k \rightarrow+\infty} k^{-1} \sup _{j \in \mathbb{N}} \sum_{i=j}^{k+j-1} m^{-1} g_{m}\left(\theta^{i m} x\right),
$$

which proves (16). Moreover, by Theorem 4.5(ii), we have

$$
\limsup _{m \rightarrow+\infty} \sup _{k \in \mathbb{N}} m^{-1} g_{m}\left(\theta^{k m} x\right) \leq \limsup _{m \rightarrow+\infty} m^{-1} \sup _{u \in \mathbb{N}} g_{m}\left(\theta^{u} x\right)=\Sigma(x),
$$

which proves (17) and completes the proof of (iii).

R e m a r ks. In [8, pp. 116-117], formulae are given for central and singular exponents of a non-autonomous linear differential equation in $\mathbb{R}^{d}$, which can be easily derived from the continuous parameter version of Theorem 5.17 (see Section 6).

6. Continuous parameter case. The results of the present paper can be generalized to the continuous parameter case. We say that $\mathcal{G}:=\left(g_{t}\right)_{t \in \mathbb{R}^{+}}$ is a continuous subadditive process if $(x, t) \rightarrow g_{t}(x)$ is continuous and $g_{t+s} \leq$ $g_{t}+g_{s} \circ \theta^{t}$ for all $s, t \in \mathbb{R}^{+}$, where $\Theta:=\left(\theta^{t}\right)_{t \in \mathbb{R}^{+}}$is a semiflow on $X$. We adapt the other definitions from the discrete case with necessary changes. There are two methods of deriving continuous parameter versions of the results given in the preceding sections.

Firstly, we can adapt the theorems and proofs from the discrete case replacing Cesàro means by integral means. Secondly, we can use the following method.

Let $T \in \mathbb{R}^{+}-\{0\}$. Then $\left\{\theta^{n T}: n \in \mathbb{N}\right\}=: \Theta_{T}$ is a discrete semidynamical system and $\left\{g_{n T}: n \in \mathbb{N}\right\}=: \mathcal{G}_{T}$ is a discrete c.s.p. over $\Theta_{T}$. Its exponents are related to those of $\mathcal{G}$ in the following way.

THEOREM 6.1. Let $\mathcal{G}:=\left(g_{t}\right)_{t \in \mathbb{R}^{+}}$be a c.s.p. such that there exists a c.s.p. conjugate to $\mathcal{G}$ and satisfying condition $(\beta)$, and let $T \in \mathbb{R}^{+}-\{0\}$. Then

(i) for every non-empty compact invariant set $A$, there exists $C \in \mathbb{R}^{+}$ such that for all $t \in \mathbb{R}^{+}$,

$$
\left\|\frac{1}{t} g_{t}-\frac{1}{[t / T] T} g_{[t / T] T}\right\|_{A} \leq \frac{C}{t},
$$

(ii) for all characteristic, central, singular and global exponents, the exponent for the continuous process $\mathcal{G}$ is equal to the corresponding exponent for the discrete process $\mathcal{G}_{T}$ divided by $T$. 
Proof. Let $t, T \in \mathbb{R}^{+}$. Put

$$
C_{T}:=\max \left\{\max \left(g_{s}(x), h_{s}(x)\right): x \in A \text { and } 0 \leq s \leq T\right\},
$$

where $\mathcal{H}:=\left(h_{n}\right)_{n \in \mathbb{R}^{+}}$is a c.s.p. conjugate to $\mathcal{G}$ and satisfying $(\beta)$. Then

$$
\left|g_{[t / T] T}(x)-g_{t}(x)\right| \leq C_{T} \quad \text { and } \quad\left|g_{[t / T] T}(x)\right| \leq[t / T] C_{T} .
$$

Hence we obtain the required formula with $C=2 C_{T}$. The assertion (ii) follows from (i), Theorems 4.1, 4.3, 4.5 and their continuous parameter counterparts, which can be proved by the first method.

The above theorem has twofold applications. Firstly, putting $T=1$, we can use it to derive continuous parameter results from their discrete parameter counterparts. Secondly, taking $T \neq 1$, we can obtain new formulae for exponents. This theorem applies in particular to c.s.p.'s generated by cocycles with values in $\mathrm{Gl}(d)$ (by Proposition 5.8) and to c.s.p.'s generated by cocycles with values in $\{M \in M(d): M$ has strictly positive entries $\}$ (by Propositions 5.9 and 5.10).

Acknowledgments. I am especially indebted to Dr. Marek Capiński for introducing me to the subject of Lyapunov exponents, for his critical reading of the manuscript and for many valuable suggestions. Moreover, I wish to express my gratitude to Professor David Elworthy and the SERC Foundation for making possible my stay at the University of Warwick during which this work was begun. I would also like to thank Professor Elworthy for his kind encouragement and for many preliminary talks. Finally, I am grateful to Professors Andrzej Lasota, Anthony Manning, Michał Misiurewicz and Bolesław Szafirski for many helpful discussions and suggestions.

\section{References}

[1] H. D. I. A barbanel, R. Brown and M. B. Kennel, Variation of Lyapunov exponents on a strange attractor, J. Nonlinear Sci. 1 (1991), 175-199.

[2] J. C. Alexander and J. A. Yorke, Fat baker's transformations, Ergodic Theory Dynamical Systems 4 (1984), 1-23.

[3] G. Benettin, L. Galgani and J.-M. Strelcyn, Kolmogorov entropy and numerical experiments, Phys. Rev. A 14 (1976), 411-418.

[4] G. Benettin, L. Galgani, A. Giorgilli and J.-M. Strelcyn, Lyapunov characteristic exponents for smooth dynamical systems; a method for computing all of them, Part 1: Theory, Meccanica 15 (1980), 9-20; Part 2: Numerical application, ibid., 21-29.

[5] R. Bowen and D. Ruelle, The ergodic theory of Axiom A flows, Invent. Math. 29 (1975), 181-202.

[6] L. A. Bunimovich, Statistical properties of Lorenz attractors, in: Nonlinear Dynamics and Turbulence, G. I. Barenblatt, G. Iooss and D. D. Joseph (eds.), Pitman, Boston, Mass., 1983. 
[7] L. A. Bunimovich and Ya. G. Sina ̌, Stochasticity of the attractor in the Lorenz model, in: Nonlinear Waves, A. V. Gaponov-Grekhov (ed.), Nauka, Moscow, 1979, 212-226.

[8] B. F. Bylov, R. È. Vinograd, D. M. Grobman and V. V. Nemytskiǔ, Theory of Lyapunov Exponents, Nauka, Moscow, 1966 (in Russian).

[9] A. Carverhill, Flows of stochastic dynamical systems: Ergodic theory, Stochastics 14 (1985), 273-317.

[10] P. Constantin and C. Foiaş, Global Lyapunov exponents, Kaplan-Yorke formulas and the dimension of the attractors for $2 D$ Navier-Stokes equations, Comm. Pure Appl. Math. 38 (1985), 1-27.

[11] H. Crauel, Lyapunov exponents and invariant measures of stochastic systems on manifolds, in: Lyapunov Exponents, Proceedings, Bremen 1984, L. Arnold and V. Wihstutz (eds.), Lecture Notes in Math. 1186, Springer, 1986, 271-291.

[12] N. Dunford and J. T. Schwartz, Linear Operators, Part I, Wiley, New York, 1958.

[13] J. P. Eckmann and D. Ruelle, Ergodic theory of chaos and strange attractors, Rev. Modern Phys. 57 (1985), 617-656.

[14] A. Eden, Local Lyapunov exponents and a local estimate of Hausdorff dimension, RAIRO Modél. Math. Anal. Numér. 23 (1989), 405-413.

[15] - Local estimates for the Hausdorff dimension of an attractor, J. Math. Anal. Appl. 150 (1990), 100-119.

[16] J. D. Farmer, Chaotic attractors of an infinite-dimensional dynamical system, Phys. D 4 (1982), 366-393.

[17] J. D. Farmer, E. Ott and J. A. Yorke, The dimension of chaotic attractors, ibid. 7 (1983), 153-180.

[18] P. Frederickson, J. L. Kaplan, E. D. Yorke and J. A. Yorke, The Liapunov dimension of strange attractors, J. Differential Equations 49 (1983), 185-207.

[19] C. Froeschle, The Lyapunov characteristic exponents and applications, J. Méc. Théor. Appl. Numéro spécial (1984), 101-132.

[20] H. Furstenberg, Recurrence in Ergodic Theory and Combinatorial Number Theory, Princeton Univ. Press, Princeton, 1981.

[21] C. Grebogi, E. Ott and J. A. Yorke, Crises, sudden changes in chaotic attractors, and transient chaos, Phys. D 7 (1983), 181-200.

[22] M. R. Herman, Construction d'un difféomorphisme minimal d'entropie topologique non nulle, Ergodic Theory Dynamical Systems 1 (1981), 65-76.

[23] R. A. Johnson, Ergodic theory and linear differential equations, J. Differential Equations 28 (1978), 23-34.

[24] R. A. Johnson, K. J. Palmer and G. Sell, Ergodic properties of linear dynamical systems, SIAM J. Math. Anal. 18 (1987), 1-33.

[25] Yu. Kifer, Characteristic exponents of dynamical systems in metric spaces, Ergodic Theory Dynamical Systems 3 (1982), 119-127.

[26] —, Ergodic Theory of Random Transformations, Birkhäuser, Boston, 1986.

[27] J. F. C. Kingman, The ergodic theory of subadditive stochastic processes, J. Roy. Statist. Soc. Ser. B 30 (1968), 499-510.

[28] -, Subadditive ergodic theory, Ann. Probab. 1 (1973), 883-909.

[29] —, Subadditive processes, in: Ecole d'Eté de Probabilités de Saint-Flour V-1975, Lecture Notes in Math. 539, Springer, 1976, 167-223.

[30] U. Krengel, Ergodic Theorems, de Gruyter, Berlin, 1985.

[31] F. Ledrappier, Some relations between dimension and Lyapounov exponents, Comm. Math. Phys. 81 (1981), 229-238. 
[32] R. Mañé, Oseledec's theorem from the generic viewpoint, in: Proceedings Internat. Congress Math., Warszawa 1983, PWN, Warszawa, 1984, 1269-1276.

[33] V. M. Millonshchikov, Typical properties of conditional exponential stability $I V$, VIII, Differentsial'nye Uravneniya 20 (1984), 241-257, 1366-1376 (in Russian); English transl.: Differential Equations 20 (1984), 187-201, 1005-1014.

[34] V. I. Oseledec, A multiplicative ergodic theorem. Lyapunov characteristic numbers for dynamical systems, Trudy Moskov. Mat. Obshch. 19 (1968), 179-210 (in Russian); English transl.: Trans. Moscow Math. Soc. 19 (1968), 197-231.

[35] K. R. Parthasarathy, Probability Measures on Metric Spaces, Academic Press, New York, 1967.

[36] M. S. Ranghunathan, A proof of Oseledec's multiplicative ergodic theorem, Israel J. Math. 32 (1979), 356-362.

[37] D. Ruelle, Ergodic theory of differentiable dynamical systems, Inst. Hautes Études Sci. Publ. Math. 50 (1979), 275-306.

[38] - Characteristic exponents and invariant manifolds in Hilbert space, Ann. of Math. 115 (1982), 243-290.

[39] S. H. Saperstone, Semidynamical Systems in Infinite Dimensional Spaces, Springer, New York, 1981.

[40] M. A. Shereshevsky, Lyapunov exponents for one-dimensional cellular automata, J. Nonlinear Sci. 2 (1992), 1-8.

[41] I. Shimada and T. Nagashima, A numerical approach to ergodic problem of dissipative dynamical systems, Progr. Theoret. Phys. 61 (1979), 1605-1616.

[42] W. Słomczyński, Subadditive ergodic theorems in $C(X)$, Riv. Mat. Pura Appl., to appear

[43] R. Temam, Infinite-Dimensional Dynamical Systems in Mechanics and Physics, Springer, New York, 1988.

[44] P. Thieullen, Entropy and the Hausdorff dimension for infinite-dimensional dynamical systems, J. Dynamics Differential Equations 4 (1992), 127-159.

[45] R. È. Vinograd, On the central characteristic exponent of a system of differential equations, Mat. Sb. 42 (84) (1957), 207-222 (in Russian).

[46] P. Walters, Unique ergodicity and random matrix products, in: Lyapunov Exponents, Proceedings, Bremen 1984, L. Arnold and V. Wihstutz (eds.), Lecture Notes in Math. 1186, Springer, 1986, 37-55.

[47] A. Wolf, J. B. Swift, H. L. Swinney and J. A. Vastano, Determining Lyapunov exponents from a time series, Phys. D 16 (1985), 285-317.

INSTITUTE OF MATHEMATICS

JAGIELLONIAN UNIVERSITY

REYMONTA 4

30-059 KRAKÓW, POLAND 